\title{
Noise characteristics of the Escherichia coli rotary motor
}

Diana Clausznitzer ${ }^{1,2,3}$ and Robert G Endres ${ }^{1,2^{*}}$

\begin{abstract}
Background: The chemotaxis pathway in the bacterium Escherichia coli allows cells to detect changes in external ligand concentration (e.g. nutrients). The pathway regulates the flagellated rotary motors and hence the cells' swimming behaviour, steering them towards more favourable environments. While the molecular components are well characterised, the motor behaviour measured by tethered cell experiments has been difficult to interpret.

Results: We study the effects of sensing and signalling noise on the motor behaviour. Specifically, we consider fluctuations stemming from ligand concentration, receptor switching between their signalling states, adaptation, modification of proteins by phosphorylation, and motor switching between its two rotational states. We develop a model which includes all signalling steps in the pathway, and discuss a simplified version, which captures the essential features of the full model. We find that the noise characteristics of the motor contain signatures from all these processes, albeit with varying magnitudes.

Conclusions: Our analysis allows us to address how cell-to-cell variation affects motor behaviour and the question of optimal pathway design. A similar comprehensive analysis can be applied to other two-component signalling pathways.
\end{abstract}

\section{Background}

Biological systems sense stimuli from their environment using cell-surface receptors, and process this information to make reliable decisions, e.g. where to move, or whether to divide or to express new enzymes. Typically, intracellular signalling molecules are activated by modification, e.g. phosphorylation or methylation, and interact in complicated biochemical reaction networks. The biochemical reactions in such networks rely on probabilistic collisions of a limited number of molecules. Hence, the number of signalling molecules fluctuates with time, making signal processing noisy. The abundance of noise sources in a cell is in stark contrast to the remarkable accuracy with which cells are known to respond to minute amounts of chemical concentration, including growing axons and immune cells $[1,2]$.

The high biological relevance of noise has widely been recognised and studied extensively in gene expression [3-7]. In contrast, noise in signal transduction is less

\footnotetext{
* Correspondence: r.endres@imperial.ac.uk

'Division of Molecular Biosciences, Imperial College London, SW7 2AZ London, UK

Full list of author information is available at the end of the article
}

well characterised, despite its importance for accurate sensing and cell-decision making. Examples of eukaryotic systems, in which signalling noise has been considered include the ultrasensitive thresholding cascades $[8,9]$, pheromone sensing in yeast $[10,11]$, signal transduction in photoreceptors [12], feedback loops for noise suppression [13,14], and eukaryotic chemotaxis [15]. Furthermore, signalling noise has been considered in parts of bacterial pathways [16-19]. However, such analyses have either been not comprehensive, or signal and noise transmission have not been compared in detail with experimental measurements along the pathway. An important class of signalling pathways are the bacterial two-component systems, including hundreds of pathways responsible for wide ranging functions such as sensing of and responding to nutrients, osmolarity, antibiotics, as well as quorum signals [20]. A particularly well characterised example is the chemotaxis pathway in E. coli (Figure 1), allowing cells to swim towards nutrients and away from toxins with high sensitivity over a wide range of ambient concentrations [21-25]. Specifically, the kinase CheA autophosphorylates when receptors are active and passes on phosphoryl groups to the 


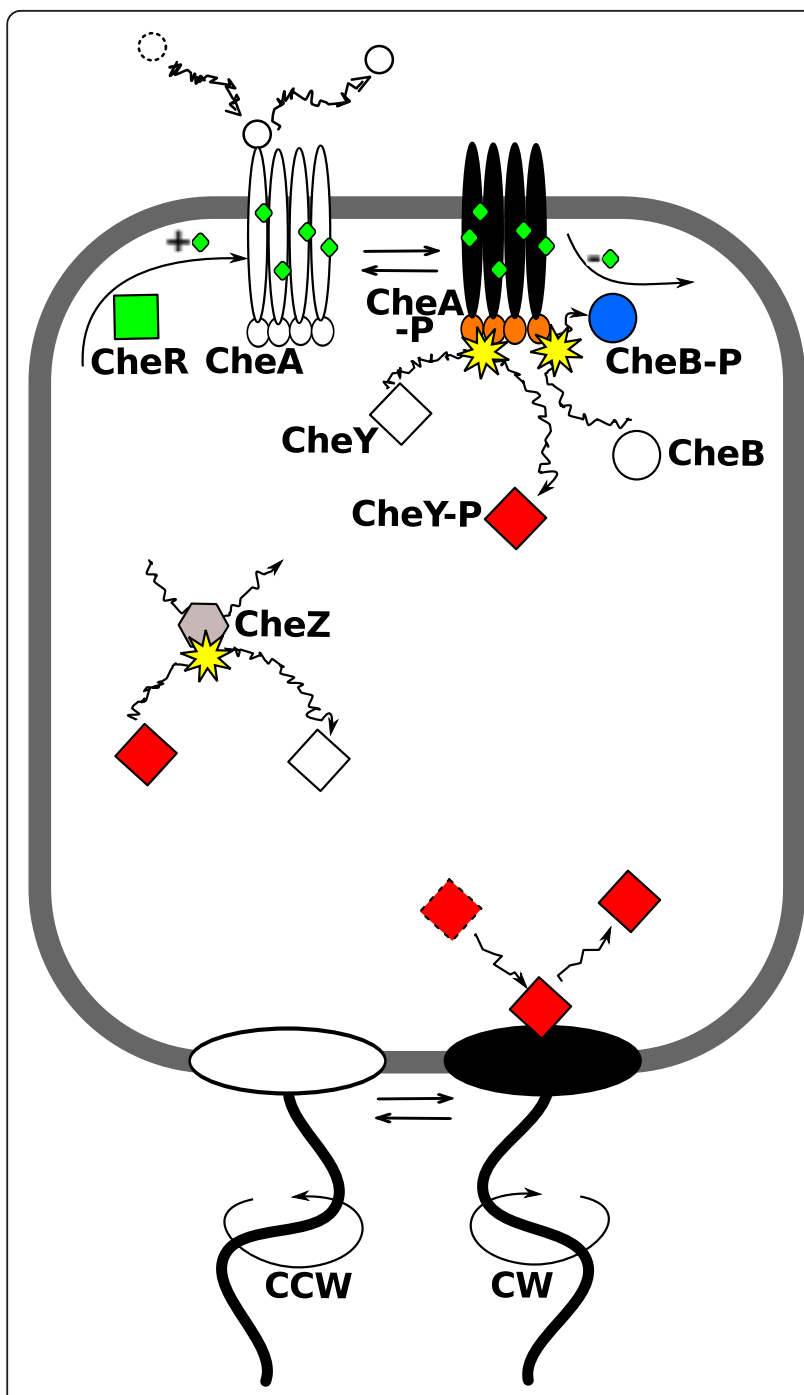

Figure 1 Chemotaxis pathways of $E$. coli. The pathway consists of transmembrane chemoreceptors, which constantly undergo molecular transitions between their on (black) and off (white) conformations. Receptors signal to CheA, which subsequently autophosphorylates. The average receptor activity is modulated by ligand binding and unbinding, as well receptors methylation and demethylation. CheA phosphorylates the response regulator CheY, which diffuses through the cell and binds to the rotary motors. Upon CheY-P binding, motors switch from their default state of CCW rotation (i.e. running mode) to $\mathrm{CW}$ rotation (i.e. tumbling mode). In addition, CheY-P is dephosphorylated by its phosphatase CheZ. Receptor methylation is catalysed by CheR, which preferentially modifies inactive receptors. Receptor demethylation is catalysed by CheB, which is activated by phosphorylation, and modifies preferentially active receptors.

response regulators $\mathrm{CheY}$ and $\mathrm{CheB}$. Phosphorylated CheY (CheY-P) modulates the probability of counterclockwise $(\mathrm{CCW})$ or clockwise $(\mathrm{CW})$ rotation of the motor. The rotational directions of motors correspond to the two swimming modes of the bacterium, namely smooth swimming and tumbling, respectively. Adaptation, i.e. the reversal of the effect of changes in the ligand concentration, is mediated by reversible receptor methylation and demethylation, catalysed by enzymes CheR and phosphorylated CheB (CheB-P), respectively. Using the E. coli chemotaxis pathway as an example, we are interested in the behaviour of the rotary motor, i.e. the cell's final output, and how its rotation is affected by signalling and noise.

To characterise signal propagation along the pathway, we use the so-called linear response function. This function characterises the response to a small time-varying input signal, such as impulse, sinusoidal and step stimuli. However, knowing the linear response function allows the calculation of system response to arbitrary input signals, provided they are sufficiently small (see Figure 2 and Methods). Typically, any system is subject to various sources of noise, i.e. random fluctuations in the input, as well as from signal processing. This is true in particular for biological systems, which rely on biochemical reactions and conformational changes of signalling molecules, which are intrinsically probabilistic. Here, we use the noise power spectrum to characterise fluctuations. The power spectrum captures the correlations between fluctuations in motor behaviour at different time points (see Figure 2 and Methods). In order to make the analysis easier, we typically consider the Fourier transforms of the dynamical equations of our model. The Fourier transform is an equivalent description of time-dependent data, and represents its decomposition into its frequency components. Using this analysis, there are several fundamental questions we would like to address:

Firstly, what types of signals are transmitted and what types are attenuated by the pathway? Early work showed that the system responds to the time-derivative of the input signal [26]. A number of research groups have measured the averaged response of cells to chemotactic signals [26-28], and found that slowly, as well as rapidly changing input signals are not transmitted by the pathway. The response to slowly changing signals is attenuated by adaptation, which reverses the activation by ligand binding [28-30]. Rapidly changing signals were conjectured to be attenuated by a third-order filter [26,27]. While the phosphorylation dynamics of CheY-P has been shown to contribute a first-order filter [30], the exact filtering dynamics of the full pathway has not been addressed.

Secondly, how is noise generated, amplified or filtered in the signalling pathway, and how do different sources of noise affect the motor behaviour? The power spectrum of the motor rotation was measured for wild-type cells and mutant cells lacking the chemotaxis signalling pathway [31]. The spectrum was found to have a large low-frequency component in the wild-type cells, indicating that there is a dominant noise source in the signalling pathway with long correlations. In simulations of the signalling 


\section{Signal}

\section{Input}
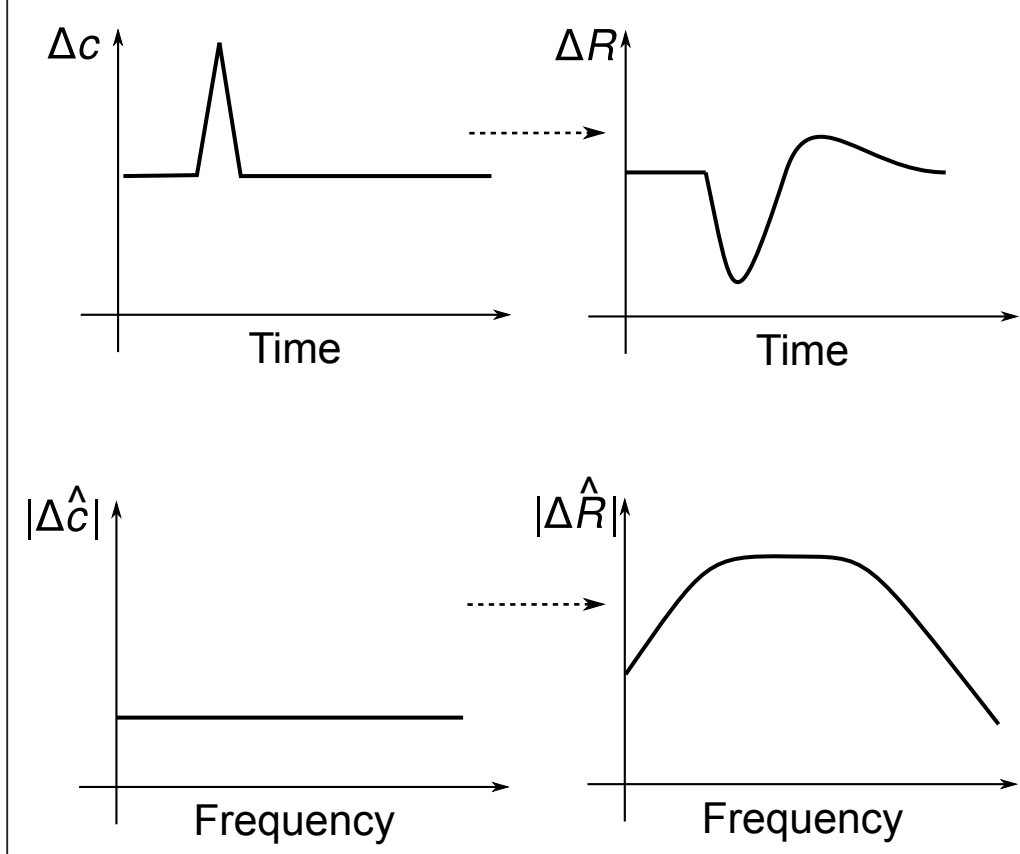

Noise

\section{Output}
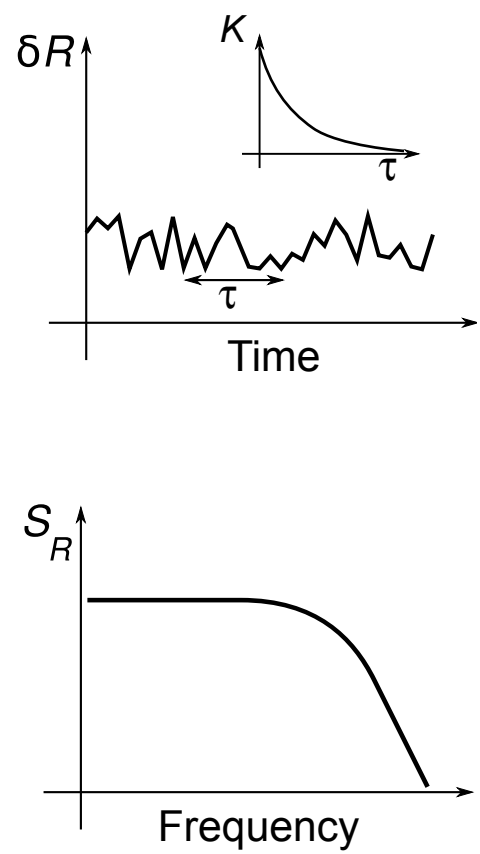

Figure 2 Characterisation of signal and noise propagation. Signal response and fluctuations can be analysed in the time domain or frequency domain, the latter allowing for analytical treatment. Analysis of signal propagation: A small stimulus $\Delta c(t)$ (Input) is applied, which results in a measurable response $\Delta R(t)$ (Output). The response $\Delta R(t)$ of the system to an impulse input represents the linear response function $\chi_{R}$ ( $t$ ) (up to a constant factor). In the frequency domain, this stimulus is a constant. The Fourier transformed linear response function

$\Delta \hat{R} \propto \hat{\chi}_{R}(\omega)$ can be analysed for its frequency-resolved transmission behaviour. Noise propagation: Fluctuations are characterised by their correlations over the time interval $\tau$. The autocorrelation function $K(\tau)$ (Inset) typically decreases as a function of interval length. In the frequency domain, the noise power spectrum $S_{R}(\omega)$, which is the Fourier transform of the autocorrelation function, characterises the frequency components of the noise.

pathway, the adaptation dynamics can play an important role in generating long correlations [16,31]. However, only signalling up to CheY-P was considered. Other studies include stochastic simulations of the noisy biochemical reactions of the pathway [32], and addressed the mechanism of motor rotation [33-37], including the thermodynamics of motor switching [38-40]. However, noise generation, filtering and amplification has not been addressed systematically for the various levels of the signalling pathway from chemoreceptors to motors.

Finally, how reliably are concentration input signals transmitted through the pathway in the presence of noise? An important task for the cell is to generate an appropriate motor response to input signals in the presence of fluctuations in the input, as well as due to noise in the biochemical signalling pathway. Furthermore, cell-to-cell variation in protein expression influences signal transmission and noise filtering. Comparing these two aspects of the pathway dynamics, namely chemotactic signal transmission and noise filtering, in close comparison with experimental data along the pathway is a novel perspective in our present study.

In the following, we present a mathematical model for the chemotaxis signalling pathway. A simplified pathway is discussed in the text to provide intuitive understanding. However, results are shown for an extended model for the full pathway provided in the Methods section. We discuss the average (deterministic) response of the signalling pathway to concentration signals. We analyse the noise sources in the signalling pathway and their effects. Finally, we vary pathway parameters and study how they affect signal and noise transmission. We also discuss briefly how our approach can be applied to other two-component systems and signalling pathways.

\section{Results}

Experimental measurements of response and noise spectrum

The signal propagation in the chemotaxis pathway has been characterised by the response to small 
concentration signals (linear response function; see Methods). Specifically, the response has been measured at the level of CheY-P using fluorescence resonance energy transfer (FRET) by Shimizu et al. [28]. In that study the system was stimulated by a periodic variation of the concentration of attractant $\alpha$-methyl-DL-aspartate (MeAsp). Using a series of frequencies of the stimulation, the magnitude (modulus) and phase, i.e. the lag between signal and response, of the response was determined. In cell-tether experiments of motor rotation, the response to short impulses of attractants was measured at the level of the motor by Block et al. [26] and Segall et al. [27]. Such data determines the linear response function up to a constant factor. Experimental results are shown in Figure 3. Noise propagation in wild-type cells has only been studied at the level of the motor by Korobkova et al. [31]. We use the experimental response functions to calibrate our model, and subsequently study the noise power spectrum and signal-to-noise ratio.

\section{Simplified model for the pathway}

Here we consider a simplified pathway to gain intuition of the key processes involved. The simplified pathway consists of chemoreceptor signalling in response to ligand binding and receptor methylation, as well as the rotary motor. Specifically, we use stochastic differential equations in a Langevin approach [41] to describe the dynamics of each type of signalling protein. We assume throughout that fluctuations in concentration are small, allowing us to describe the average behaviour of a signalling molecule by a deterministic dynamics and the fluctuations around the mean by additive noise.

We assume $N$ receptors form cooperative signalling complexes, which can switch between an active (on) and an inactive (off) state. Their activity $A$ is described by the Monod-Wyman-Changeux (MWC) model [42-47]. The activity depends on the external ligand concentration $c$ at the receptor complex, as well as the methylation level $M$ of the complex as detailed in Methods.

We consider $N_{C}$ receptor complexes in a cell, and assume that each complex signals independently of the others. The total activity $A_{c}$ of all receptors in a cell is determined by the sum over all signalling complexes $j$. The dynamics of the total activity is

$$
\frac{d A_{c}}{d t}=\sum_{j=1}^{N_{C}} \frac{\partial A}{\partial M} \frac{d M_{j}}{d t}+\frac{\partial A}{\partial c} \frac{d c_{j}}{d t}+\eta_{A_{j}}(t),
$$

i.e. the dynamics of the complex activity is affected by changes in the receptor complex methylation level (first
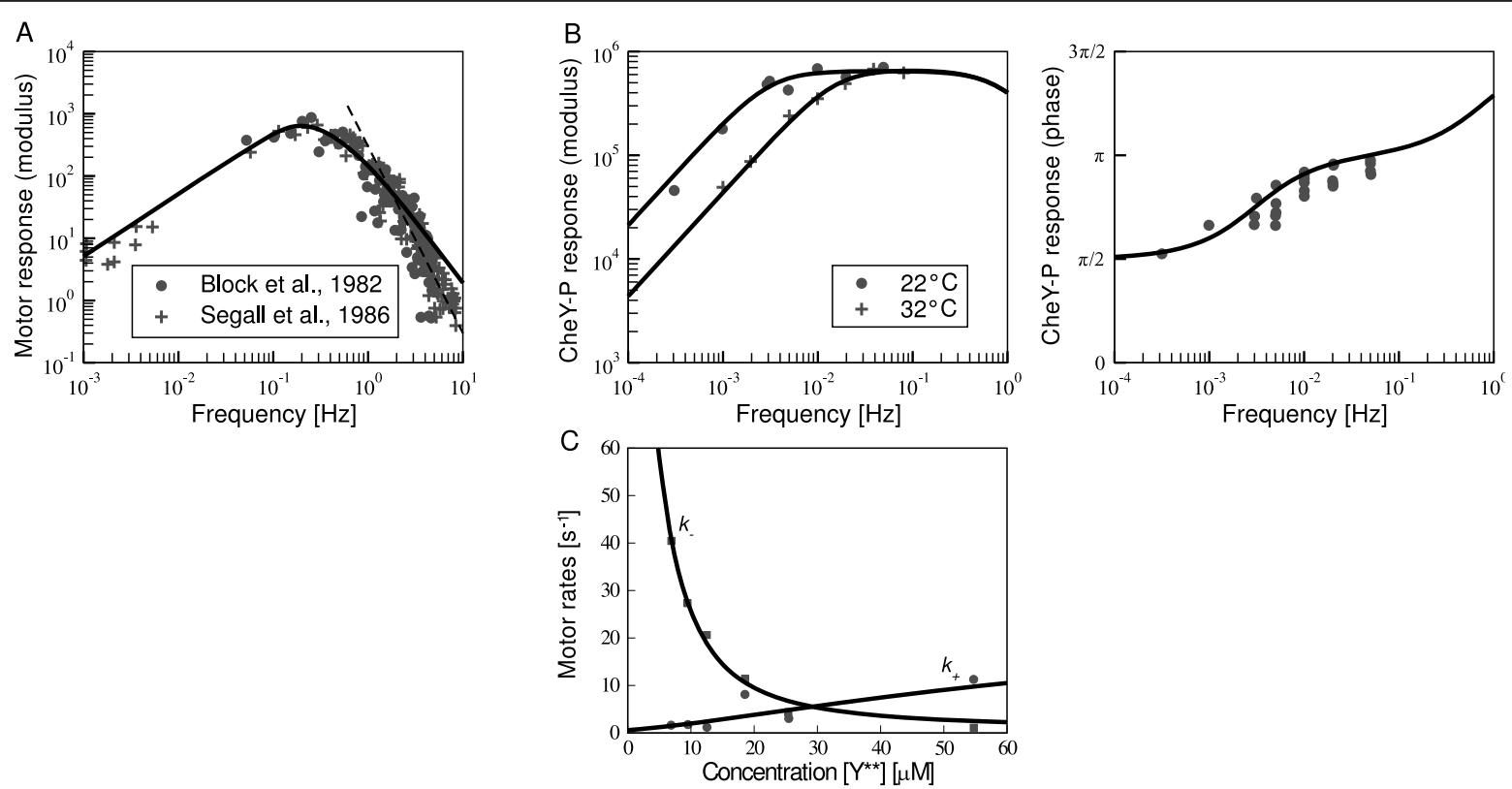

Figure 3 Calibration of the model. (A and B) Fourier transformed linear response function. (A) Magnitude of the response function of the rotary motor measured by Block et al. [26] (circles) and Segall et al. [27] (plus symbols). The fit of our model is shown by the solid line. The dashed line represents a 3rd-order filter for comparison. (B) Magnitude (left) and phase (right) of the response function at the level of the response regulator $\mathrm{CheY}$ measured by Shimizu et al. [28]. The symbols are measurements at $22^{\circ} \mathrm{C}$ (circles) and $32^{\circ} \mathrm{C}$ (plus symbols). Grey lines represent the fit of our model to the magnitude of the response. (C) Switching rates of the motor from CCW to CW rotation $k_{+}$(squares) and from CW to CCW rotation $k_{-}$(circles) as a function of the concentration of signalling-active unphosphorylated double-mutant $Y^{* *}$. The cellular concentration of $Y^{* *}$ varied as a result of induced expression in cells in which wild-type CheY and other cytoplasmic chemotaxis proteins had been deleted. A fit using the model of Turner et al. [39] is shown as well (solid and dashed lines; cf. Methods). 
term), changes in ligand concentration (second term), as well as fluctuations due to the switching of the complex between its states (last term). All noise terms $\eta(t)$ introduced in this section are discussed in Methods.

Changes in the concentration originate from timevarying input signals $\langle c(t)\rangle$, as well as fluctuations due to ligand diffusion. The dynamics of the concentration at the $j$ th receptor complex is given by

$$
\frac{d c_{j}}{d t}=\frac{d\langle c(t)\rangle}{d t}+\eta_{c_{j}}(t)
$$

where the first term captures average concentration changes (indicated by angular brackets $\langle\ldots .\rangle$.$) , affecting all$ receptors, and the second term describes concentration fluctuations at each receptor complex, assumed to be uncorrelated between different receptor complexes.

Adaptation is provided by reversible receptor methylation and demethylation, whose dynamics is described by the following equation [47]:

$$
\frac{d M_{j}}{d t}=\gamma_{R}\left(N-A_{j}\right)-\gamma_{B} A_{j}^{3}+\eta_{M_{j}}(t) .
$$

The total methylation level $M_{j}$ of a receptor complex $j$ is changed by methylation of receptors in the inactive state by CheR (first term) and demethylation (second term). This latter rate is assumed to be strongly dependent on the receptor complex activity as only active receptors are demethylated by phosphorylated demethylation enzymes CheB-P. These may act cooperatively, in agreement with time course data of wild-type and a CheB adaptation mutant [47]. The last term represents fluctuations due to the noisy processivity of the methylation and demethylation enzymes.

The motor is described as a two-state system with $\mathrm{CW}$ and $\mathrm{CCW}$ rotating states, corresponding to running and tumbling modes, respectively. The dynamics of the motor bias $X$ is derived from the Master equation of an ensemble of two-state motors, and is given by

$$
\frac{d X}{d t}=k_{+}\left(A_{c}\right)(1-X)-k_{-}\left(A_{c}\right) X+\eta_{X}(t),
$$

with $X$ the fraction of motors in the CW rotational state and applying the single-motor limit. In Eq. 4, the first term represents the switching from CCW to CW with the transition rate $k_{+}$, the second term represents switching from $\mathrm{CW}$ to $\mathrm{CCW}$ with transition rate $k$, and the third term describes temporal fluctuations in switching rates due to the stochastic nature of motor switching. Note that the Langevin equation for the two-state motor is exact [48], see also Additional File 1.

Here, transition rates are modulated by the receptor signalling activity $A_{c}$, whereas in the full pathway model CheY-P modulates motor switching. These rates have been experimentally measured using signalling mutants expressing varying amounts of constitutively active signalling molecule CheY [39]. The switching rates, including a fit of the model we used [39] (cf. Methods) to the data, are shown in Figure 3C.

\section{Signal propagation}

We consider the response to input concentration signals at various levels in the signalling pathway to study how signals are transmitted to the rotary motor (see Meth$o d s)$. Briefly, an input signal $\Delta c(t)$ is a concentration change relative to a constant background concentration $c_{0}$, affecting all receptors equally and representing a "meaningful" input to the chemotaxis signalling pathway. Hence, the concentration is given by $\langle c(t)\rangle=c_{0}+$ $\Delta c(t)$. Furthermore, cells are assumed to be adapted to the pre-stimulus concentration $c_{0}$ with the various levels $R$ of the signalling pathway adapted to their steady-state values $R^{*}$.

\section{Analytical results for linear response functions}

We can analytically calculate the Fourier transformed linear response function from the dynamical equations Eq. 1-4 without noise (see Methods). We can analyse the filtering of the signal at each level of the pathway. The Fourier transformed linear response function for the total activity of all receptors in a cell is

$$
\hat{\chi}_{A_{c}}(\omega)=\frac{-i \omega N_{C} \frac{\partial A}{\partial c}}{\omega_{M}-i \omega}
$$

The receptor activity is a high-pass filter: The magnitude of the response function is small for frequencies $\omega$ below $\omega_{M}=\left(\gamma_{R}+3 \gamma_{B} A^{* 2}\right) \partial A / \partial M$, which is the characteristic frequency due to adaptation. For frequencies above $\omega_{M}$ the response function is a constant, given by the number of receptor complexes $N_{C}$ participating in the response, and their sensitivity $\partial A / \partial c$ to ligand, evaluated at steady-state. The sensitivity is proportional to the receptor complex size $N$, i.e. it describes the amplification of the response of a single receptor.

Similarly, the Fourier transformed response of the motor is given by

$$
\hat{\chi}_{X}(\omega)=\frac{\omega_{2}}{\omega_{X}-i \omega} \hat{\chi}_{A_{c}}(\omega)
$$

The motor is a low-pass filter, i.e. its dynamics introduces a frequency-dependent response, which is constant below the characteristic frequency $\omega_{X}=k_{+}^{*}+k_{-}^{*}$ of the motor due to the steady-state switching rates $k_{+}^{*}$ and $k_{-}^{*}$. The parameter $\omega_{2}$ describes the sensitivity of motor switching with respect to changes in receptor activity (Methods). At frequencies above $\omega_{X}$ the response is reduced. From Eq. 6 it is obvious that receptors and motor are in a cascade: The motor response introduces 
a new filter proportional to $\left(\omega_{i}-i \omega\right)^{-1}$ which simply multiplies the response function of the response of the receptor activity. The response functions of the full pathway including the phosphorylation reactions are shown in Additional File 1.

For further analysis, we can write the Fourier transformed linear response function as

$$
\hat{\chi}_{R}(\omega)=\left|\hat{\chi}_{R}(\omega)\right| e^{i \phi_{R}(\omega)},
$$

where $\left|\hat{\chi}_{R}\right|$ is the magnitude and $\varphi_{R}$ is the phase of the response function, which characterise the amplitude and lag of the response behind the input signal, respectively.

\section{Model calibration}

Figure 3 shows experimental data for the response function, as well as the fits of our full pathway model. Block et al. [26] and Segall et al. [27] measured the response of the motor using impulses of attractant. For our fit we adjusted adaptation and motor switching rates. Compared to the data by Shimizu et al. [28] at the same temperature, adaptation rates are one order of magnitude larger, i.e. adaptation is faster in these experiments. The parameter $\omega_{X}$ of the motor switching is $2.1 / \mathrm{s}$, consistent with switching rates of about $1 \mathrm{~Hz}$ [29]. It is not clear from where the difference in adaptation rates between the two sets of experiments originates. However, different strains and media can lead to large variations in receptor expression level [49]. Besides different experimental conditions, Shimizu et al. [28] used populations of cells, whereas measurements by Segall et al. [27] were done on single cells. For the fit of our model to the data by Shimizu et al. [28], we adjusted only the adaptation rates, as measurements were restricted to low frequencies. The fit at $32^{\circ} \mathrm{C}$ yields the same adaptation parameters as obtained from fitting dose-response curves of adapting cells [47] (Figure 3B, left). The adaptation rates for room temperature are one order of magnitude smaller. Importantly, fitting to the magnitude of the Fourier transformed response yields a good fit for the phase of the response as well (Figure 3B, right). Fitted parameters are given in the Methods.

\section{Signal filtering along the pathway}

Figure 4 shows simulated time courses of the chemotactic response to an concentration impulse and the Fourier transforms of corresponding linear response functions, as well as our analytical results. As can be seen in the figure, linear response functions for the numerically solved non-linear model indeed match the analytically calculated functions, confirming that our calculation results are valid for the chosen input signal. We observe how the input signal is transmitted through the pathway, with the effective pulse durations becoming progressively longer along the pathway (Figure 4, left), including total receptor activity in a cell $\left(A_{c}\right)$, phosphorylated kinase CheA, phosphorylated response regulator $\mathrm{CheY}$, and finally the motor $(X)$. In Figure 4, middle we show the corresponding linear response functions.

The receptor activity acts as a high-pass filter, i.e. it transmits high-frequency signals, but not low-frequency signals. As can be seen from our simple model (cf. Eq. 5 ), this property is due to adaptation, which introduces the time-derivative of the signal $\Delta c(t)$ up to the characteristic frequency $\omega_{M}$, eliminating the response to slowly changing attractant concentrations. The activity of chemoreceptors is the input to further levels in the pathway. The response of CheA-P is fast, and shows no qualitative difference to the response of receptors in the frequency range shown. In contrast, due to the fast but finite rates of phosphorylation and dephosphorylation, preventing the CheY-P concentration to respond to rapidly changing input signals, the response at the level of $\mathrm{CheY}$ is reduced at high frequencies. Similarly, the motor introduces another high-frequency filter due to slow switching between its two states. This additional filter can be deduced from Eq. 6, where the motor response function takes the response of chemoreceptors as input, and additionally introduces a characteristic cut-off frequency $\omega_{X}$ due to slow motor switching rates. Hence, the chemotaxis pathway acts as a band-pass filter [26], which only transmits input signals within a selected frequency range, which is of the order of 1 to $10 \mathrm{~s}$. This time scale corresponds to the average time between two tumbles, allowing sensing of concentration changes during periods of running. As shown in Figure 4 , middle the phase tends towards $\pi / 2$, i.e. a quarter period, at low frequencies. This has been analysed only for the receptor complex activity [28]. This phase difference is due to adaptation and represents the fact that the system takes the time derivative of the stimulus below the characteristic frequency $\omega_{M}$ of adaptation. The phase shift of the receptor activity increases to $\pi$ at high frequencies, indicating that the activity simply follows the output (a negative sign is due to the negative response of the activity to attractant concentration [28]). The phase at high frequencies for the response of CheA follows the phase of the receptor activity, except for a small increase of the phase shift. In contrast, the phase of CheY and the motor increase significantly beyond $\pi$ indicating that slow rates of modification and motor switching introduce a lag of the response behind the stimulus.

\section{Noise propagation}

To understand the noise characteristics of the motor, we consider the noise sources and their transmission in the pathway. Each step in the signalling pathway is essentially probabilistic, hence, noisy: ligand diffusion 


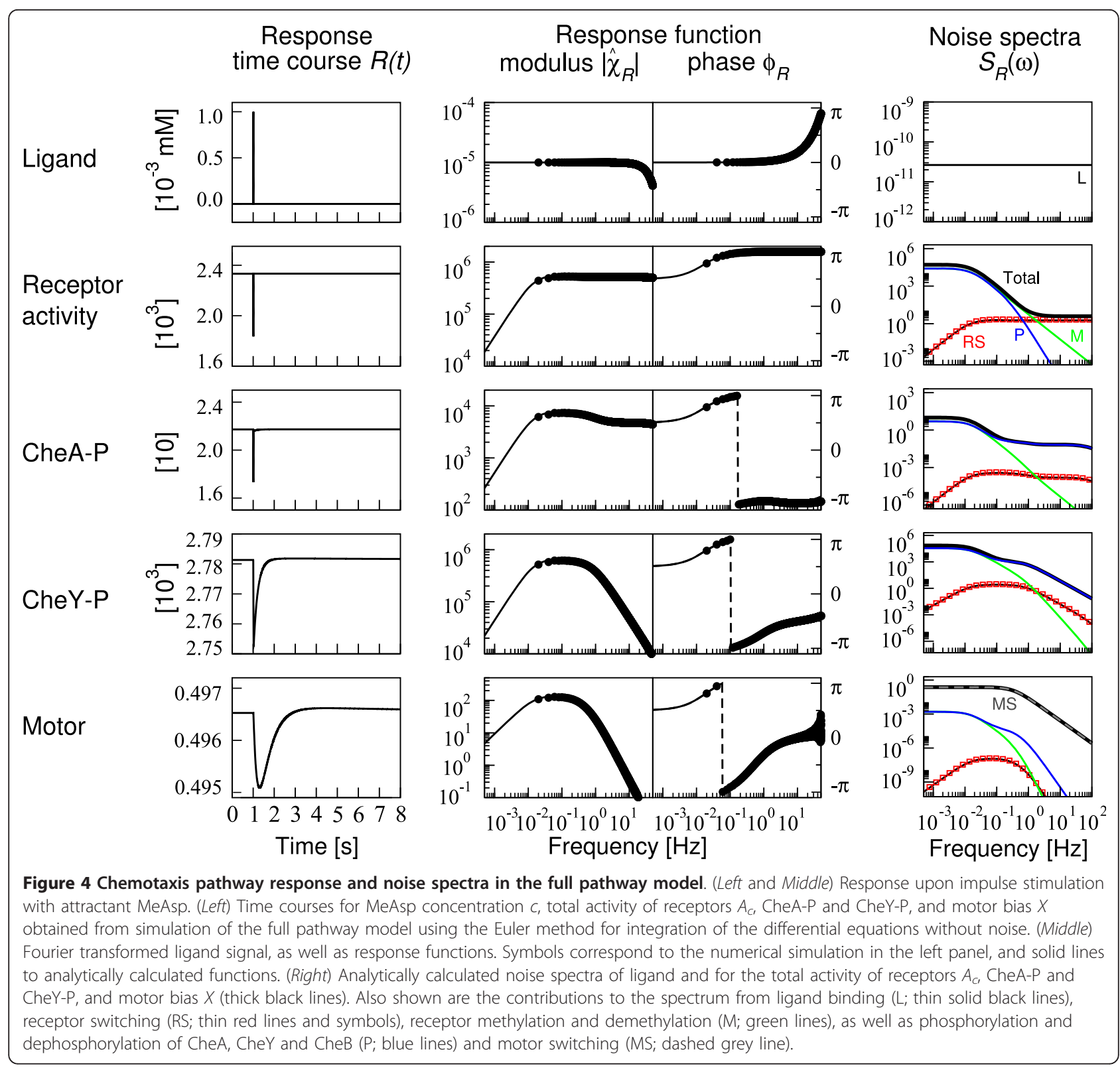

and binding, receptor switching between its functional on and off states, as well as receptor methylation and demethylation, phosphorylation and dephosphorylation of signalling proteins $\mathrm{CheA}$, CheY and $\mathrm{CheB}$, and switching of the rotary motor between its two states, CW and CCW rotation. To characterise fluctuations of the phosphorylated signalling protein $\delta R(t)$ around its mean value $\langle R(t)\rangle$, we use the power spectrum $S_{R}$ $(\omega)$ and the variance $\left\langle\delta R^{2}\right\rangle=\left\langle R^{2}(t)\right\rangle-\langle R(t)\rangle^{2}$ (cf. Methods).

\section{Analytical results for noise spectra}

Considering Eq. 1-4 with noise, we can analytically calculate power spectra (see Methods). The power spectrum of activity fluctuations is given by

$$
S_{A_{c}}(\omega)=N_{C} \frac{\omega^{2}\left[S_{a}(\omega)+\left(\frac{\partial A}{\partial c}\right)^{2} S_{c}(\omega)\right]+\left(\frac{\partial A}{\partial M}\right)^{2} Q_{M}}{\omega_{M}^{2}+\omega^{2}} .
$$

In this equation we considered fluctuations from receptor switching (first term in numerator), ligand diffusion (second term), as well as the receptor methylation dynamics (third term) at each of the $N_{C}$ receptor complexes per cell. We have assumed that fluctuations at different receptor complexes are independent. Therefore, we obtain the sum of $N_{C}$ identical spectra for all complexes. The individual terms $S_{a}(\omega), S_{c}(\omega)$ and $Q_{M}$ are given by Eq. 30, 34 and 36 in Methods. The frequency dependence of the ligand noise, as well as noise 
from receptor complex switching, indicates filtering of slowly varying fluctuations with frequencies below the characteristic frequency $\omega_{M}$ due to adaptation. In contrast, only high-frequency fluctuations from the receptor methylation dynamics are filtered by the adaptation dynamics. This is due to finite rates of methylation and demethylation fluctuations introducing correlations in the receptor methylation level.

The power spectrum of fluctuations in the motor bias is obtained as

$$
S_{X}(\omega)=\frac{\omega_{2}^{2} S_{A_{c}}(\omega)+Q_{X}}{\omega^{2}+\omega_{X}^{2}} .
$$

The first term represents transmitted noise from receptor complexes, including the noise power spectrum of the receptor activity and the sensitivity $\omega_{2}^{2}$ of motor switching rates to changes in activity. The second term is motor switching noise. Both noises are filtered by the motor, as its finite rates of switching introduce correlations with characteristic frequency $\omega_{X}$. The noise spectra of the full pathway including the phosphorylation reactions are shown in Additional File 1.

\section{Noise filtering along the pathway}

In Figure 4, right we show the power spectrum of fluctuations at the various levels of the signalling pathway, i. e. total receptor activity, CheA-P, CheY-P and the motor. We also plot the individual contributions from processes generating noise, namely ligand diffusion, receptor switching, methylation and demethylation of receptors, and phosphorylation and dephosphorylation of proteins, as well as motor switching. This allows us to follow how noise is generated and transmitted at the various levels of the pathway. The noise spectrum of the receptor activity has its largest contribution at low frequencies, which originates in the receptor methylation and phosphorylation dynamics. Most of the fluctuations from phosphorylation stem from $\mathrm{CheB}$ (the separate contributions to the phosphorylation noise are not shown in Figure 4, right). At high-frequencies, the activity noise spectrum is at. This is due to ligand and receptor switching noise, which is removed at low frequencies by adaptation, but not at high-frequencies. The general behaviour of the noise spectrum corresponds to the simplified model (cf. Eq. 8).

The noise spectrum of CheA-P has generally the same shape as the activity spectrum with a large low-frequency component, mainly due to receptor methylation and $\mathrm{CheB}$ phosphorylation dynamics. This spectrum also has an almost flat high-frequency behaviour in the frequency range shown. Apart from ligand and receptor switching noise, the flat part of the spectrum is largely determined by fluctuations from CheA autophosphorylation, which has roughly the same shape as activity noise at high frequencies because autophosphorylation depends on the receptor activity.

The noise spectrum of CheY-P is also largest at low frequencies. However, at high frequencies the spectrum falls off as noise is filtered due to the finite rates of CheY phosphorylation and dephosphorylation, which introduce correlations in the fluctuations.

The motor introduces another layer of filtering of transmitted noise with the characteristic motor switching frequency $\omega_{X}$ (cf. Eq. 9). Hence, transmitted noise is reduced by two filters in the frequency range shown, namely due to the CheY-P and motor dynamics. However, the main contribution to the spectrum is due to the motor switching itself, which is reduced only by a first-order filter with characteristic frequency $\omega_{X}$.

\section{Cell-to-cell variation of motor behaviour}

How are the signal response, fluctuations and the signalto-noise ratio (SNR) affected by changing parameters of the pathway such as size of receptor complexes, protein concentrations and reaction rate constants? In this section, we discuss the effect of cell-to-cell variation on the power spectrum of the motor. In the next section, we discuss the SNR and its contributions, and how they depend on receptor complex size and adaptation rates.

According to our model parameters obtained from fitting the Fourier transformed linear response to data, the main contribution to the power spectrum comes from the steady-state switching of the motor between $\mathrm{CCW}$ and CW state. However, cell-to-cell variation in protein content and motor switching rates can lead to modifications of the largely Lorentzian-shaped spectrum. These modifications are caused by the transmitted noise from receptor methylation and phosphorylation dynamics (green and blue lines in Figure 4, right). Specifically, Figure $5 \mathrm{~A}$ shows the motor power spectrum for increased motor switching rates as well as reduced adaptation rates and number of chemoreceptors in a cell. In all cases the low-frequency component of the transmitted noise becomes more prominent.

An increased low-frequency component has been observed in the motor power spectrum for cells with low motor bias [31]. Both, wild-type cells and mutants lacking the signalling pathway were measured. Hence, the mutant's spectrum represents the component to the power spectrum from steady-state motor switching only. Wild-type cells showed a large low-frequency component compared to the mutants. Figure 5B shows that our model can reproduce these experimental data (shown in the Inset), provided we assume a low CCW to $\mathrm{CW}$ switching rate leading to small motor bias (see Methods for the details of parameters). Specifically, the low-frequency component of our spectrum originates from noise in the methylation and phosphorylation 

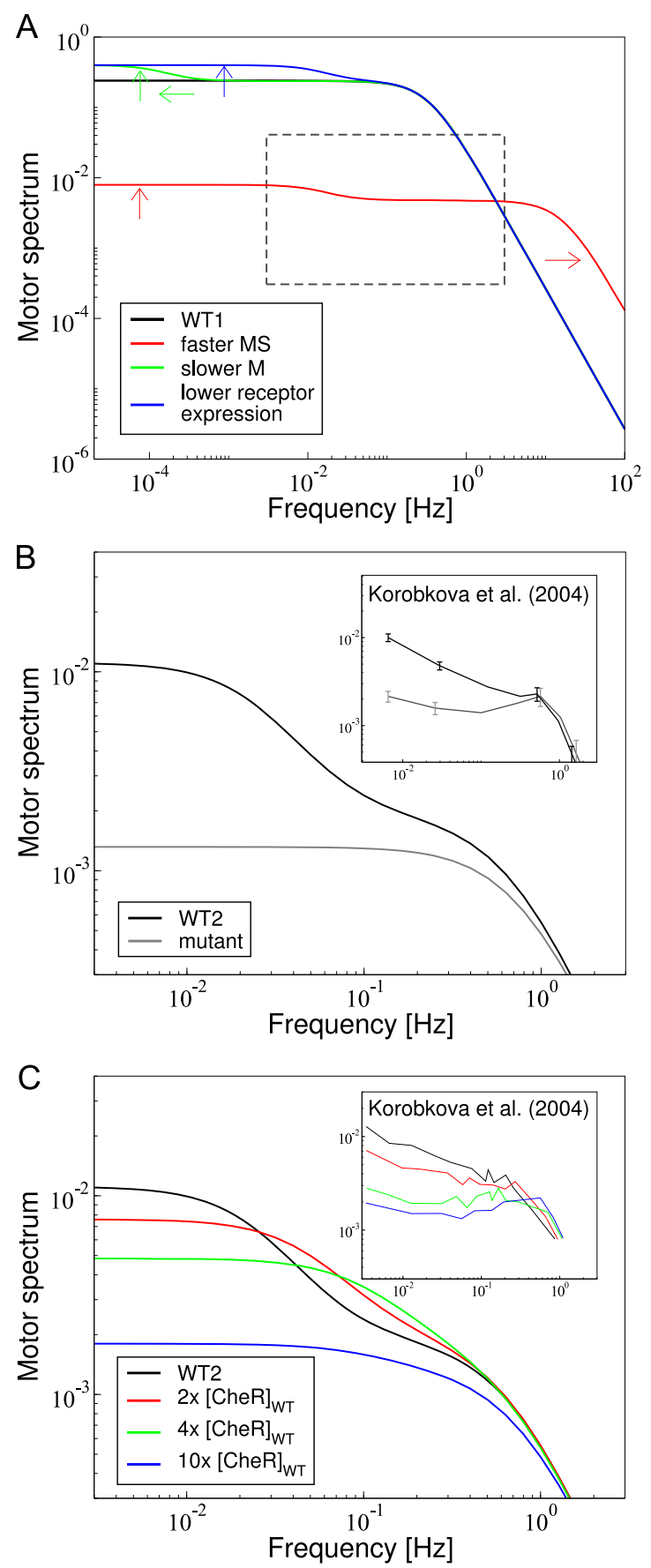

Figure 5 Effect of cell-to-cell variation on power spectrum of the motor in the full pathway model. (A) Predictions about how different cell parameters affect the motor power spectrum, in particular its low-frequency component. The black line (wild-type WT1) is the same as the total motor spectrum in Fig. 4, right. The motor spectra for increased motor switching rates (MS; red line), as well as reduced rates of receptor methylation and demethylation ( $\mathrm{M}$; green line) and the total number of receptors (blue line) are shown as well. Arrows indicate the features in the spectra that are affected. Dashed box is the area shown in panel (B). (B) Motor spectrum of cells with low motor bias (black line), as well as the component from steady-state motor switching only (grey line).
(Inset) Measured power spectra for wild-type cells (WT2) with low motor bias (black) and mutant lacking the signalling pathway (grey). Axes are the same as in the main panel. Error bars indicate the measurement uncertainty. Spectra were traced from data presented by Korobkova et al. [31]. Model parameters are listed in the Methods. (C) Motor spectrum of cells with varying CheR expression level. Shown are WT2 (black line; same as in panel B), as well as 2(red), 4-(green) and 10-fold (blue) CheR-expression level compared to wild-type. (Inset) Measured power spectra of cells with corresponding CheR-expression level (see main panel). Axes are the same as in the main panel. Spectra were traced from data presented by Korobkova et al. [31]. Model parameters are listed in the Methods.

dynamics in cells with low motor bias. Furthermore, experiments show that the low-frequency component in the motor power spectrum is reduced by increasing the expression level of CheR [31]. In Figure $5 \mathrm{C}$ we show that our model reproduces this experimental finding (shown in the Inset). The low-frequency component due to noise from receptor methylation and demethylation is effectively reduced by increasing the methylation rate constant.

\section{Signal-to-noise ratio at the motor}

To characterise how signals are transmitted in the presence of noise, we define the SNR at the level of the motor as

$$
S N R=\frac{\Delta X^{2}}{\left\langle\delta X^{2}\right\rangle}
$$

with $\Delta X^{2}$ and $\left\langle\delta X^{2}\right\rangle$ defined in Methods. For optimal signalling this ratio should be maximised. For simplicity, we only discuss the receptor activity in the text, while in the figures we additionally show the contribution from phosphorylation processes as transmitted to the motor in the full pathway model.

\section{Optimal receptor complex size}

Receptor complexes amplify small signals proportionally to their size $N$. However, also concentration fluctuations are expected to be amplified. Hence, we hypothesise that the receptor complex size could be optimised to yield a balance of advantageous amplification of signals and detrimental amplification of input noise.

In Figure 6A we show the integrated motor response $\Delta X^{2}$ (see Methods) to a step stimulus for varying background concentration and receptor complex size. We assume that the step stimulus size is a constant fraction of 10 percent of the background concentration. The integrated response has a characteristic variation with background concentration with the maximum in the sensitivity range of Tar receptors (indicated by their dissociation constants). Furthermore, the response increases with receptor complex size $N$. We calculated 


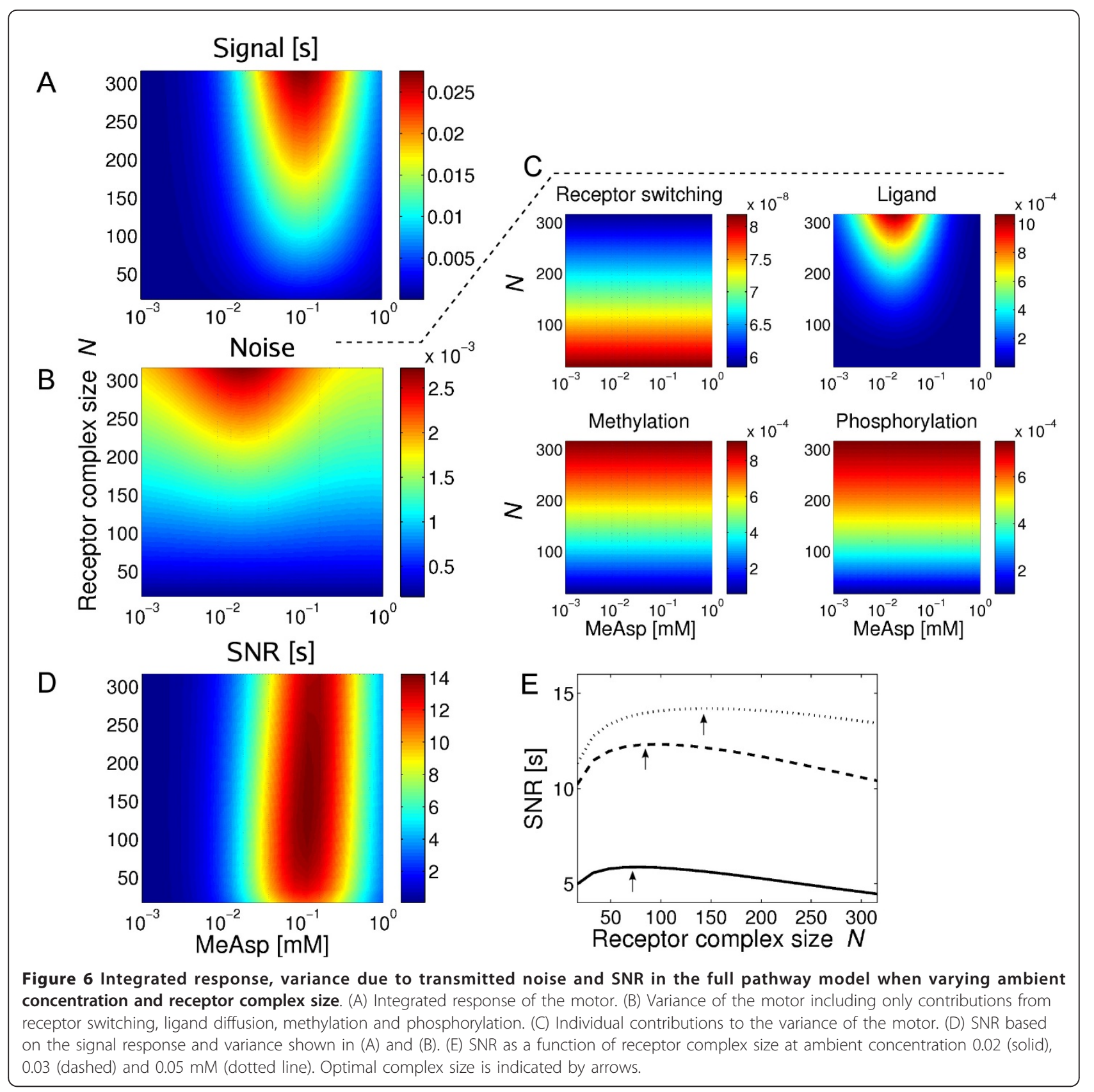

the integrated signal response of the receptor activity (see Methods). This quantity scales linearly with receptor complex size, $\Delta A_{c}^{2} \propto N$, due to coherent addition of the signalling responses of different receptor complexes, amplification of concentration changes by receptor complexes, as well as filtering by adaptation.

In Figure 6B and 6C, we show the variance (i.e., the integrated noise power spectrum, see Methods) of the transmitted noise of the pathway at the level of the motor. Only the contribution to the variance from ligand diffusion depends on the background concentration. Compared to the signal response, the maximum of the variance is shifted to a slightly lower concentration. The contribution to the variance from switching of receptor complexes is relatively small compared to the other contributions and roughly constant with receptor complex size, whereas those from ligand diffusion, receptor methylation and phosphorylation dynamics increase with receptor complex size.

To understand these behaviours of the variance more intuitively, we analysed the receptor activity analytically (for details of the calculation, see Methods). We find, the contribution to the variance of the receptor activity from receptor switching is indeed constant, independent 
of $N$. The contribution from ligand diffusion scales steeply as $N^{2}$, the difference between ligand noise and ligand signal amplification being due to $(i)$ noise from different complexes is added up incoherently, and (ii) the main contribution to the variance coming from high-frequency ligand noise, which is not filtered by adaptation. The contribution from receptor methylation grows approximately linearly with receptor complex size as a result of the incoherent addition of fluctuations at different receptor complexes and the sensitivity of the receptor complex activity with respect to changes in methylation level increasing proportionally with $N$. The contribution to the variance from phosphorylation processes grows with receptor complex size similar to the contribution from the methylation dynamics. Overall, the total variance of transmitted noise at the level of the motor has contributions from receptor switching, the dynamics of receptor methylation, and phosphorylation. The latter is approximately constant or grows slower than the amplified signal response, whereas the component from ligand diffusion increases steeper than the signal response with growing receptor complex size.

The resulting SNR, i.e. the ratio of integrated signal response and variance of the noise, is shown in Figure $6 \mathrm{D}$ and $6 \mathrm{E}$. The SNR is largest at background concentrations in the sensitivity range of the Tar receptor. Furthermore, due to the different dependencies of the signal and the noise on the receptor complex size, the SNR has a maximum at a particular receptor complex size (Figure 6E). The SNR grows below that complex size due to signal amplification, while the amplified ligand noise from ligand diffusion is still below the internal noise level from receptor switching and receptor methylation and phosphorylation dynamics. Above the optimal receptor complex size, the SNR decreases because the ligand noise is amplified more than the signal.

\section{Optimal adaptation rates}

As shown above, adaptation filters slow input signals, with the adaptation speed determining what input frequencies are transmitted by the pathway. Furthermore, the adaptation dynamics filters input noise. Hence, adaptation rates may be expected to be optimised for signal and noise propagation.

Figure 7A shows the integrated signal response at the level of the motor for varying rates of receptor methylation $\left(\gamma_{R}\right)$ and demethylation $\left(\gamma_{B}\right)$. Varying these parameters describes changing the concentrations of receptor modification enzymes $C h e R$ and $C h e B$. The integrated signal response is found to be maximal when both rates of methylation and demethylation become small simultaneously. Then, the adapted receptor activity is in the steep region of dose-response curves, and the initial response to attractant is maximal. At the same time adaptation becomes slow and, therefore, the response lasts long. Interestingly, varying the two parameters independently has different effects on the signalling response: the integrated signal response increases for vanishing $\gamma_{R}$, whereas it decreases for vanishing $\gamma_{B}$. There are two effects that contribute to this behaviour, illustrated in Figure 7B: Firstly, if the concentration of one of the receptor modification enzymes is reduced, the receptors becomes modified predominantly by the opposing enzyme, hence driving the receptor activity towards saturation $\left(A^{*}=0\right.$ or $\left.A^{*}=1\right)$. This effect would tend to quench the response by receptors. Secondly, as the enzyme concentration is reduced, adaptation times increase. Hence, this effect increases the integrated signal response as the time the receptor activity deviates from the adapted state increases. According to calculations shown in Methods for the integrated response of receptors, the first effect dominates in the case of reduced $\gamma_{B}$ : Due to the strong activity dependence of the demethylation rate, reducing the demethylation rate constant effects the adapted activity of receptors strongly. Hence, receptors are quickly driven into saturation for vanishing $\gamma_{B}$. In contrast, in the case of reduced $\gamma_{R}$ the second effect dominates and the increased adaptation time leads to an increased integrated signal response. At large methylation and demethylation rates, adaptation times are reduced leading to a decreasing integrated signal response.

The variance of fluctuations is shown in Figure 7C and 7D. The individual contributions from transmitted noise at the level of the motor look qualitatively similar. For $\gamma_{R}$ and $\gamma_{B}$ such that the adapted receptor activity is in the steep region of dose-response curves, the variance of transmitted noise is largest. In contrast, for either vanishing $\gamma_{R}$ or $\gamma_{B}$ all contributions decrease, consistent with calculations for the variance of the receptor activity in Methods. In these cases, the adapted receptor activity becomes saturated, hence, quenching fluctuations transmitted by receptors.

The resulting SNR is shown in Figure 7E. The SNR increases for vanishing $\gamma_{R}$ and decreases for vanishing $\gamma_{B}$. According to Figure 7E, a large SNR is obtained for small $\gamma_{R}$ and large $\gamma_{B}$, corresponding to the parameters of our model.

\section{Fluctuation-response relationships}

Park et al. [50] presented the idea that the signalling response to concentration signals and fluctuations in the chemotaxis pathway are not independent of each other, because they are produced by the same molecular interactions. Specifically, based on measurements at the level of the motor these authors proposed a fluctuationresponse theorem, namely an approximate linear relationship between the adaptation time to step stimuli 


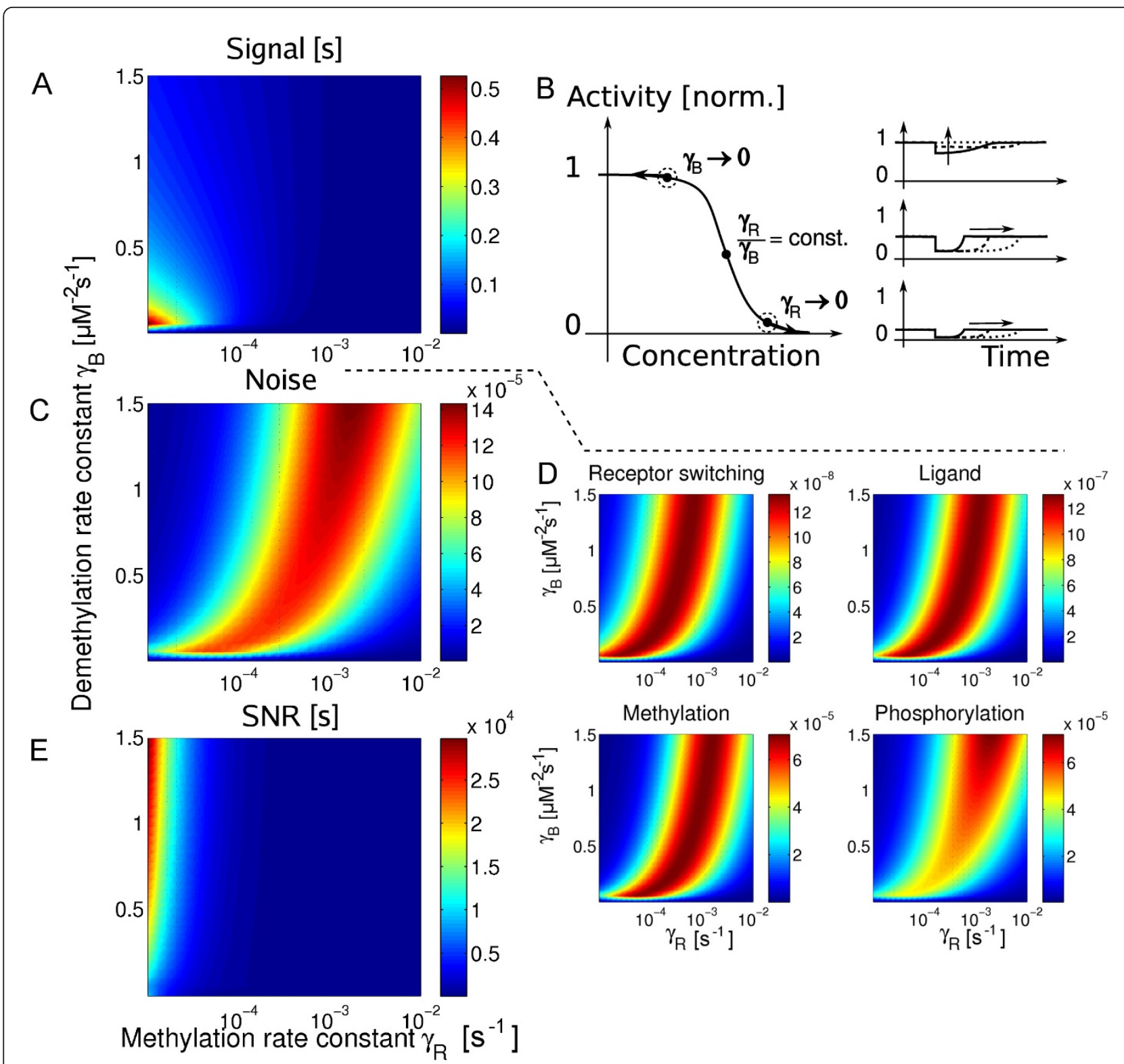

Figure 7 Integrated response, variance due to transmitted noise and SNR in the full pathway model when varying receptor methylation and demethylation rate constants $\gamma_{R}$ and $\gamma_{B}$, respectively. (A) Integrated response of the motor. (B) Illustration of the effects of vanishing $\gamma_{R}$ and $\gamma_{B}$ on adapted activity (indicated by dot and circle along dose-response curve; left), as well as on time courses (right) for three cases, $\gamma_{R} \rightarrow 0$ (top right), $\gamma_{R} / \gamma_{B}=$ const (middle right) and $\gamma_{B} \rightarrow 0$ (bottom right). For further explanation see text. (C) Variance of the motor including only from receptor switching, ligand diffusion, methylation and phosphorylation. (D) Individual contributions to the variance of the motor. (E) SNR based on the signal response and variance shown in (A) and (C).

and the variance of fluctuations in CheY-P concentration.

Using our model, we tested this hypothesis and varied the adaptation rates, as well as the total CheY concentration in a cell, resulting in a shifted adapted CheY-P concentration at steady state. We find that the variance of CheY-P (normalised by the squared adapted value) decreases as the adapted CheY-P value increases except for very small adapted CheY-P concentrations (Figure $8 \mathrm{~A}$ ), indicating that the relative strength of fluctuations decreases as expected. In Figure 8B we show the adaptation time, approximated by the inverse of the characteristic frequency due to adaptation, plotted against the variance of CheY-P. We find that at low adaptation times (thick line styles), the adaptation time increases with the variance of CheY-P, indicating that cells with 

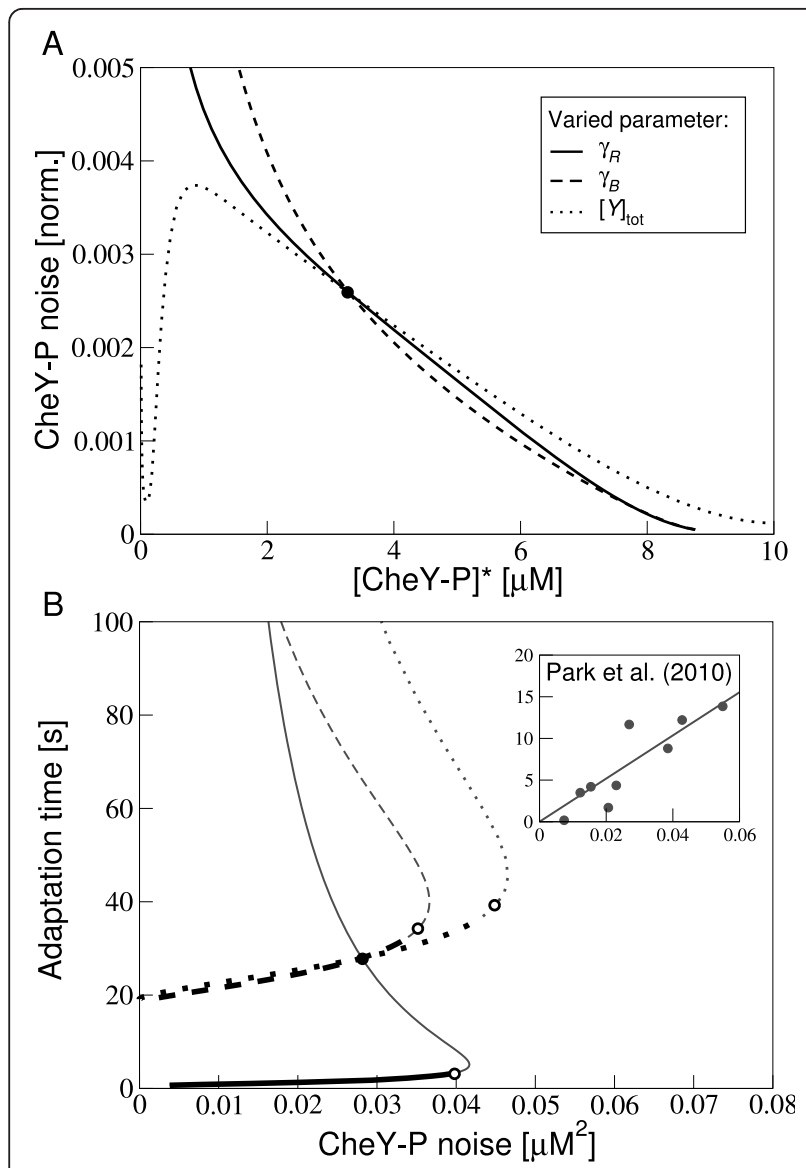

Figure 8 Fluctuation-response relationships. (A) Variance of CheY-P fluctuations (normalised by the squared adapted CheY-P value) as a function of adapted CheY-P value for varying adaptation rates $\gamma_{R}$ (solid line) and $\gamma_{B}$ (dashed line) and total CheY concentration (dotted line). The filled circle marks the point, where parameters for the three parameter variations coincide. (B) Adaptation time, calculated as $\left(\lambda_{1} \partial A / O M\right)^{-1}$, plotted against the variance of CheY-P fluctuations. Adapted CheY-P concentration of $5 \mu \mathrm{M}$ is indicated by the open circles. Legend is the same as in (A). Our parameters are given in Additional File 1. (Inset) Variance of CheY-P concentrations plotted against the adaptation time as extracted from measurements of motor rotation by Park et al. [50]. The line is a linear fit through the data.

large fluctuations also respond longer to concentration signals. In contrast at long adaptation times, the adaptation time decreases with increasing variance of the pathway (grey parts of the curves). This behaviour can be directly traced back to the non-monotonic variance shown in Figure 7. It is maximal when the adapted CheY-P concentration is about $5 \mu M$, i.e. when typically half of CheY is phosphorylated. The exact relationship depends on what parameters varied, exemplified by the different curves in Figure 8B. For each parameter and small adaptation times, we find an approximate linear relationship in line with Park et al. [50], see Inset.

\section{Discussion}

Biological signalling pathways employ biochemical reaction networks and molecular state transitions to sense and process signals from the environment. Fluctuations inherent in these processes determine the signals which can reliably be transmitted. Here, we studied the signal and noise propagation in the $E$. coli chemotaxis signalling pathway, which controls the bacterial swimming behaviour in chemical gradients. Specifically, we considered the dynamics of ligand diffusion, receptor methylation and demethylation, receptor complex switching between on and off, phosphorylation and dephosphorylation of the kinase CheA, and response regulators CheY and $\mathrm{CheB}$, as well as from rotary motor switching between $\mathrm{CW}$ and $\mathrm{CCW}$ direction. We assume cooperative chemoreceptor signalling complexes, whose activities depend on ligand concentrations and receptor methylation level, described by the MWC model [42-47].

We formulated a model which includes all processes in the signalling pathway. Not included is the dynamics of gene expression and enzyme localisation to the chemoreceptor cluster, which is assumed to be much slower than the dynamics of signalling processes (discussed below). To make results intuitive we also presented a simplified version of the model, which only includes the dynamics of the activity of chemoreceptors, ligand concentration and receptor methylation level, as well as the motor dynamics. To calibrate the model, we first collected experimental data sets for the signalling pathway and rotary motor [26-28], and the motor switching behaviour [31,51]. Using the Fourier transformed linear response function, we subsequently fitted our model parameters. We found a range of parameters fitting different data sets, revealing a striking experimental variation, which may require further characterisation in the future.

Despite the fitting, there is a discrepancy of our response function and the data at large frequencies. Block et al. [26] and Segall et al. [27] conjectured that the pathway is a third-order low-pass filter. In contrast, we find that the only relevant filters in that frequency range are due to CheY-P and motor dynamics, leading to only a second-order filter. One explanation for the missing filter is that experimental concentration pulses were not short enough, leaving a signature from the input signal at large frequencies. Alternatively, additional processes such as a slow release of CheY-P from the chemosensory complexes as discussed in Ref. [52] could lead to an additional filter. However, CheY-P/CheZ complex formation and potential oligomerisation of CheY-P/CheZ complexes [53-55] are not expected to contribute to high-frequency filtering (Additional File 1). 
The motor behaviour is the final cell output, which contains characteristic noise signatures of all upstream signalling components, including the receptors. We found that motor switching is the dominant contribution to the spectrum of the fluctuations in motor bias (Figure 5). This may be not surprising as motor switching enables $E$. coli to tumble and change its swimming direction, and is therefore crucial for its search strategy. However, low-frequency contributions from signalling and adaptation may become more significant for some cells or under certain conditions (Figure 5). Specifically, we predict that due to cell-to-cell variation of protein contents or fast motor switching, these low-frequency components become comparable to motor switching noise. For instance, Korobkova et al. [51] measured power spectra in cells with low motor bias and found that the low-frequency component is increased compared to mutants lacking the signalling pathway, and that increasing the expression level of CheR decreases the low-frequency component. Our model is able to reproduce these spectra, demonstrating the general applicability of our approach. Long correlations in motor bias may enable subpopulations of cells to swim further without tumbling or to tumble more frequently.

To test our predictions, adaptation rates can be varied using cells expressing different amounts of CheR and CheB from an inducible plasmid. Alternatively, the natural variability in protein expression between cells can be exploited. Numerous wild-type cells could be monitored. By extracting the adaptation times for chemotactic stimuli, the adaptation rate constants for individual cells can be inferred. Subsequently, the same cells can be used to measure long time traces of motor switching and noise spectra can be calculated. While we included a number of noise sources in our model, others have not been included. For instance the binding and unbinding of the adaptational enzymes to the C-termini of the receptors [56] may introduce low-frequency fluctuations in the motor noise power spectrum. However, as shown for enzyme CheR in Figure 9A, this contribution is significantly smaller than the methylation and demethylation noise. In the same plot we also show the CheR gene-expression noise, which only contributes at very low frequencies (observation times larger than $10^{3} \mathrm{~s}$ ). Furthermore, the ligand noise may be underestimated in our model since fluctuations of the ligand concentration at individual receptor complexes were treated as uncorrelated. If the fluctuations are treated as strongly correlated (on the scale of the polar chemoreceptor cluster), the ligand noise becomes substantially larger as complexes coherently transmit the ligand noise (see Figure 9B). Taken together, we believe that we included the main noise sources in our model although due to subtleties, the magnitude of noise contributions are

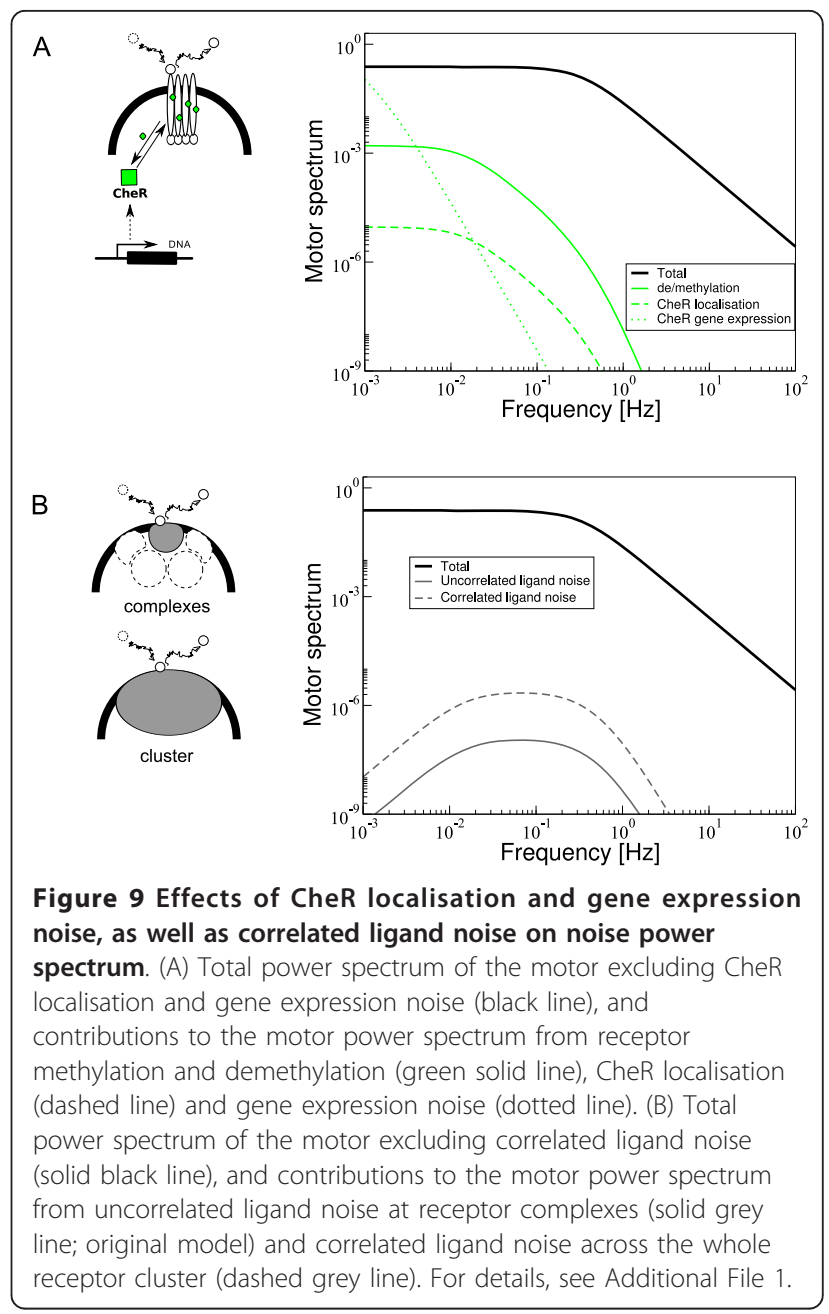

much harder to pinpoint exactly. Our full-pathway model allows us to connect to a large variety of data and literature. For instance, we also investigated how the response to concentration signals is related to fluctuations in the chemotaxis pathway (Figure 8), similar to ideas presented by Park et al. [50]. We do not find one unifying fluctuation-response theorem, but a nonlinear trend for the relationship between adaptation times and variance in CheY-P. For small adaptation times we find an approximate linear relationship in line with experiments [50].

Although chemotaxis is one of many capabilities a cell has and may not be optimised in isolation without the rest of the cell, we speculate the cell aims to maximise the SNR for most efficient signalling and chemotaxis. We found that the SNR is maximised at particular receptor complex sizes similar to values of receptor cooperativity extracted from FRET dose-response curves [46]. In line with the data, the "optimal" complex size increases with external ligand concentration, and hence with receptor methylation level. While our complex 
sizes appear overestimated, noise from ligand molecules rebinding to the same receptor complex [57] has not been considered here. This may well increase the noise level from external sources and hence decrease the predicted optimal receptor complex size. Using our model, we also analysed the effect of varying the methylation and demethylation rate constants. We found that a large SNR is obtained for small methylation and large demethylation rate constant, corresponding to our fitted model parameters from FRET dose-response curves [47].

To describe the noise, the Langevin approximation is expected to work for the phosphorylation and dephosphorylation of the abundant protein CheY. However, its applicability is less clear for receptor signalling due to both extrinsic ligand noise and intrinsic noise from receptor methylation. Furthermore, the switching of the binary motor may constitute relatively large noise. As shown in Additional File 1 the Master equation and Langevin approximation yield the same results for receptor signalling [58]. As for the motor, the Langevin equation is exact [48]. However, we explicitly tested that the statistical properties of the time series obtained for two-state switching and Langevin equation are the same. For constant rates, as well as for noisy rates due to fluctuations in CheY-P concentration, the power spectra obtained for the two processes are the same (Additional File 1). In Additional File 1 we further show that noise terms are indeed sufficiently small that linearisation of the pathway equations is justified.

\section{Conclusions}

We presented a comprehensive analysis of signal and noise propagation in the chemotaxis pathway of $E$. coli. Our full-pathway model allowed us to successfully explain a large variety of experimental data from the literature. However, our work goes beyond E. coli chemotaxis as similar methods can be applied to many other pathways.

The E. coli chemotaxis pathway is a member of the large class of two-component systems, containing hundreds of closely related pathways involved in stress response, virulence and inter-cell communication $[20,59]$. In these pathways, activation of a sensor histidine kinase results in its autophosphorylation, and subsequently in phosphorylation of a response regulator, which typically binds to DNA and regulates gene expression. The final output, i.e. activation of gene expression, is again binary and hence similar to the bacterial chemotaxis pathway (Figure 10). There are particularly well-studied examples of two-component systems: ( $i$ ) the VanS (kinase)/VanR (response regulator) system conferring vancomycin resistance in Gram positive bacteria [60], (ii) quorum sensing in Vibrio harveyi, where the three kinases LuxN, LuxQ and CqsS respond

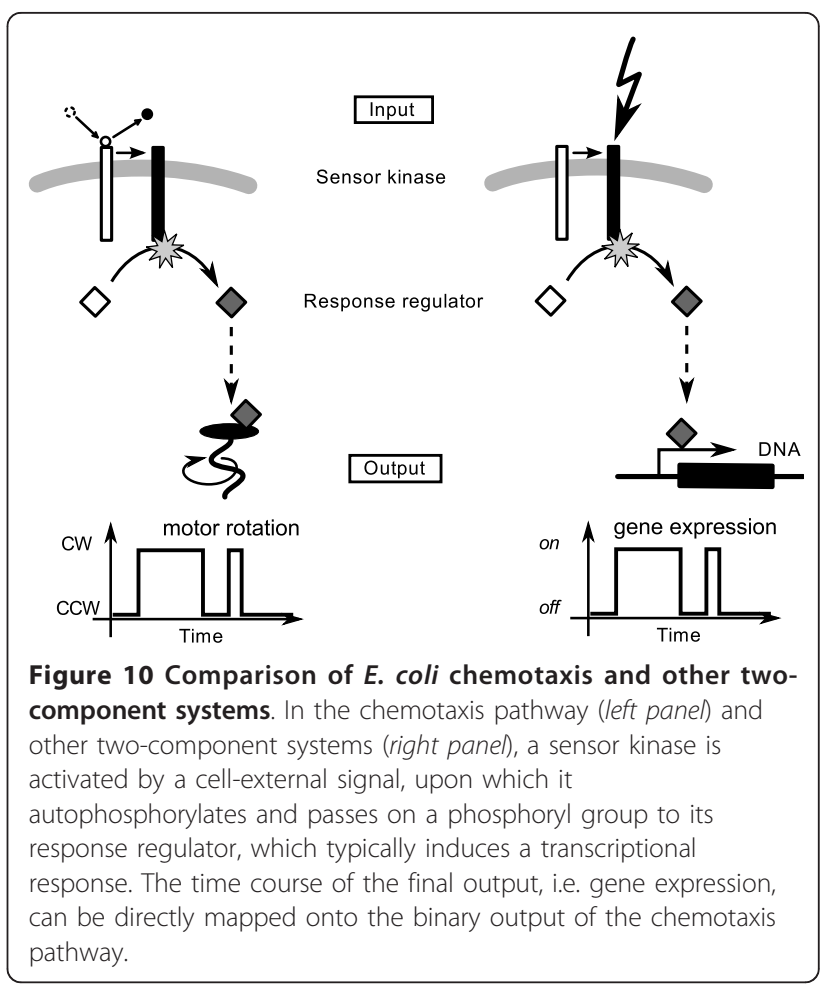

to different autoinducers and first phosphorylate the phosphotransferase LuxO (which has no equivalent in the chemotaxis pathway), which then phosphorylates the response regulator LuxU [61], and (iii) the phosphorelay controlling sporulation in Bacillus subtilis. The relay contains at least four kinases KinA-KinB and the phosphotransferase Spo0F, which phosphorylates the response regulator Spo0A [62].

Our work may also be applicable to other bacterial chemotaxis pathways, which are often considerably more complex than E. coli's pathway and still need to be understood in detail. For instance, the photosynthetic bacterium Rhodobacter sphaeroides has several homologues of each of the chemotaxis proteins in E. coli [63]. Interestingly, Rhodobacter has two chemotaxis receptor clusters, one polar cluster similar to E. coli and one cytoplasmic cluster, which is thought to sense the metabolic state of the cell. Both clusters need to be present for chemotaxis [63]. The soil bacterium Bacillus subtilis has three adaptation systems [64]: one based on methylation and demethylation of receptors similar to $E$. coli and two independent of receptor methylation, the $\mathrm{CheC} / \mathrm{CheD}$ system and the $\mathrm{CheV}$ system. Furthermore, in B. subtilis, sensory adaptation is not determined by the level of receptor methylation but the location of methylation sites on the receptors. Hence, our analysis presented here may also help elucidate the design of these pathways and clarify the computational problems these bacteria try to solve. 


\section{Methods}

\section{MWC model for activity of receptor complexes}

The MWC model describes signalling by receptor complexes, which can switch between their on and an off states. The average activity of a complex is given by

$$
A=\frac{N}{1+e^{F(c, M)}},
$$

ranging from zero to $N$. The free-energy difference $F$ $(c, M)$ between the on and off state is

$$
\begin{aligned}
F(c, M)= & N-\frac{1}{2} M+N\left[v_{a} \ln \left(\frac{1+c / K_{a}^{o f f}}{1+c / K_{a}^{o f f}}\right)\right. \\
& \left.+v_{s} \ln \left(\frac{1+c / K_{s}^{o f f}}{1+c / K_{s}^{o f f}}\right)\right]
\end{aligned}
$$

which is a function of the concentration $c$ present at the receptor complex site and the methylation level $M$ of the receptor complex. The methylation level of a complex is the sum of methylation levels of all receptors in a complex. Here, we consider two receptor types, Tar (indicated by index $a$ ) with fraction $v_{a}$ of receptors in the complex, and Tsr (indicated by index $s$ ) with fraction $v_{s}$ of receptors. Receptors are sensitive to attractant MeAsp with dissociation constants $K^{\text {on }}$ and $K^{\text {off }}$ in the on and off state, respectively. We use the following parameters for the MWC model for receptor complexes: $K_{a}^{\text {off }}=0.02 \mathrm{mM}, K_{a}^{o n}=0.5 \mathrm{mM}$, $K_{s}^{\text {off }}=100 \mathrm{mM}$ and $K_{s}^{\text {on }}=10^{6} \mathrm{mM}[44,47]$.

\section{Stochastic differential equations for full pathway model}

Equations 1-4 represent a simplified model of the chemotaxis pathway to illustrate signalling and noise transmission. In the following, our model used for the full signalling pathway is summarised. Equations 1 and 2 equally apply to the full pathway model. However, instead of Eq. 3, the dynamics of the methylation level of complex $j$ in the full pathway model is described by

$$
\begin{aligned}
\frac{d M_{j}}{d t} & =\gamma_{R}\left(N-A_{j}\right)-\gamma_{B} A_{j} B_{p}^{2}+\eta_{M_{j}}(t) \\
& =\gamma_{R}\left(N-A_{j}\right)-\frac{\gamma_{B}}{V_{\text {cell }}^{2}} A_{j} N_{B_{p}}^{2}+\eta_{M_{j}}(t)
\end{aligned}
$$

Here, we explicitly include the number of CheB-P $\left(B_{p}\right)$ molecules $N_{B_{p}}$ in the demethylation term, with $V_{\text {cell }}$ the cell volume.

In addition, we take into account phosphorylation and dephosphorylation of CheA $\left(A_{p}\right)$, CheY $\left(Y_{p}\right)$ and CheB $\left(B_{p}\right)$, which are described by the following equations:

$$
\begin{aligned}
\frac{d N_{A_{p}}}{d t}= & A_{c}\left(\frac{k_{A}}{N_{C} N}\right)\left(N_{A, t o t}-N_{A_{p}}\right)+ \\
& -\left(\frac{k_{y}}{V_{\text {cell }}}\right)\left(N_{Y, \text { tot }}-N_{Y_{p}}\right) N_{A_{p}}+ \\
& -\left(\frac{k_{b}}{V_{\text {cell }}}\right)\left(N_{B, t o t}-N_{B_{p}}\right) N_{A_{p}}+ \\
& +\eta_{A, p}(t)+\eta_{A, Y_{p}}(t)+\eta_{A, B_{p}}(t) \\
\frac{d N_{Y_{p}}}{d t}= & \left(\frac{k_{\gamma}}{V_{\text {cell }}}\right)\left(N_{Y, \text { tot }}-N_{Y_{p}}\right) N_{A_{p}}-k_{-\gamma} N_{Y_{p}}+ \\
& -\eta_{A, Y_{p}}(t)+\eta_{-Y_{p}}(t) \\
\frac{d N_{B_{p}}}{d t}= & \left(\frac{k_{b}}{V_{c e l l}}\right)\left(N_{B, t o t}-N_{B_{p}}\right) N_{A_{p}}-k_{-b} N_{B_{p}}+ \\
& -\eta_{A, B_{p}}(t)+\eta_{-B_{p}}(t)
\end{aligned}
$$

with $N_{i}$ the number of molecules of species $i$ in a cell volume. Note, that we neglected the binding of CheY-P to its phosphatase CheZ for simplicity, and describe dephosphorylation of CheY-P by the effective dephosphorylation rate $k_{-\gamma} N_{Y_{p}}$. As shown in Additional File 1 this simplification has no qualitative effect on the response function. The term $\eta_{A, p}$ describes the noise associated with CheA autophosphorylation. The terms $\eta_{A, B_{p}}(t)$ and $\eta_{A, Y_{p}}(t)$ represent the noise generated in phosphorylation of CheB and CheY by CheA, respectively. The terms $\eta_{-B_{p}}(t)$ and $\eta_{-Y_{p}}(t)$ describe the noise associated with dephosphorylation.

Note that some noise terms appear in two equations. This is due to the fact that we assign noise terms to a specific process, e.g. phosphorylation of CheY by CheA. Hence, the corresponding noise term $\eta_{A, Y_{p}}(t)$ appears in the dynamics of CheA-P and of CheY-P. As a positive fluctuation in the dynamics of CheA-P due to phosphorylation of CheY corresponds to a negative fluctuation in the dynamics of CheY-P, these noise terms appear with opposite signs in the two equations. The noise intensities and parameter values of the model are summarised below. Finally, instead of Eq. 4 the dynamics of the motor is now described in terms of

$$
\frac{d X}{d t}=k_{+}\left(N_{Y_{p}}\right)(1-X)-k_{-}\left(N_{Y_{p}}\right) X+\eta_{X}(t),
$$

where we use experimentally derived switching rates $k$ + and $k_{-}$as a function of CheY-P concentration (cf. Figure $3 \mathrm{C}$ and below).

\section{Model for motor switching rates}

Turner et al. presented a model for motor switching to explain the observed motor switching rates [39]. The 
model for motor switching is an MWC model, where 26 subunits of the motor assume one of two states corresponding to $\mathrm{CW}$ and $\mathrm{CCW}$ rotation. While these subunits bind the molecule CheY-P independently of each other, the switching of states occurs cooperatively. The authors derive the overall rates of switching (averaging over all possible CheY-P occupancy states) as

$$
\begin{aligned}
& k_{+}\left(Y_{p}\right)=k_{+}(0) \cdot\left(\frac{1+\frac{\mu Y_{p}}{K_{C C W}}}{1+\frac{Y_{p}}{K_{C C W}}}\right)^{m_{c o o p}} \\
& k_{-}\left(Y_{p}\right)=k_{-}(0) \cdot\left(\frac{1+\frac{\mu Y_{p}}{K_{C C W}}}{1+\frac{Y_{p}}{K_{C W}}}\right)^{m_{\text {coop }},},
\end{aligned}
$$

where $m_{\text {coop }}=26$ is the number of motor subunits, $-k_{B} T \ln (\mu)$ is the free-energy difference of switching per molecule of CheY-P, and $K_{C C W}$ and $K_{C W}$ are the dissociation constants for binding CheY-P in the CCW and $\mathrm{CW}$ state, respectively.

Motor switching rates $k_{+}$and $k_{-}$have been derived experimentally as a function of the concentration of a signalling mutant $\mathrm{CheY}^{* *}$, which is constitutively active [39], as shown in Figure 3C. The cellular concentration of $Y^{* * *}$ varied as a result of induced expression in cells in which wild-type CheY and other cytoplasmic chemotaxis proteins had been deleted. To obtain the switching rates in terms of CheY-P, rather than signalling mutant CheY"**, we rescaled the dissociation constants of CheY binding to the motor such that the switching rates are equal, i.e. $\mathrm{CW}$ bias about $1 / 2$, at CheY-P concentration $3.2 \mu M$ [65]. We fitted the above model to the experimental data in Figure $3 \mathrm{C}$ and the $\mathrm{CW}$ bias at $33^{\circ}$ [39] and used the rate constants $k_{+}$and $k_{-}$in our full pathway model (Eq. 17).

\section{Characterisation of signal and noise propagation Signal response}

The mean, or deterministic response of a system to an arbitrary, sufficiently small signal is described in terms of a characteristic function of the system, the linear response function $\chi_{R}$. The time-dependent response $\Delta R$ $(t)$, i.e. the deviation from the adapted state due to a small input signal $\Delta c(t)$, is linear and determined by

$$
\Delta R(t)=\int_{-\infty}^{t} \chi_{R}(t-\tau) \Delta c(\tau) d \tau
$$

Hence, the time course of the response is determined by the convolution of the linear response function and the input signal. The linear response function describes the dynamics of the pathway and the convolution with the input signal represents the fact that the current state of the system is determined by the history of the input signal [66]. Importantly, for a nonlinear system to respond linearly to a stimulus, the input signal has to be small. For the receptor complex activity, a signal is small if the change in ligand concentration is small compared to the apparent dissociation constants for the on and off states. These dissociation constants depend on the background concentration as the system adapts to the background concentration $c_{0}$, resulting in a particular free-energy difference $F^{*}$. Thus, the concentration change has to be small compared to $c_{0}+K_{a}^{o n}$ and $c_{0}+K_{a}^{o f f}$. In this case, the change in free-energy difference due to the concentration change is much smaller than $k_{B} T$. In Figure 4 , the background concentration $c_{0}$ $=0$ and the peak concentration of the impulse is $10^{-3}$ $\mathrm{mM}$, compared to $K_{a}^{o n}=0.5 \mathrm{mM}$ and $K_{a}^{\text {off }}=0.02 \mathrm{mM}$. Hence, the input signal is indeed small.

The Fourier transform of Eq. 20 reads more simply

$$
\Delta \hat{R}(\omega)=\hat{\chi}_{R}(\omega) \Delta \hat{c}(\omega) .
$$

$\hat{\chi}_{R}(\omega)$ is also called the frequency-dependent gain [12]. The modulus $\left|\hat{\chi}_{R}(\omega)\right|$ describes what frequencies of the input signal are transmitted well, and which ones are attenuated.

Typically, finite activation rates of the system limit the response to rapidly changing input signals, i.e. high-frequency signals. In this case, the Fourier transformed linear response functions falls off at high-frequencies, and the system is called a low-pass filter. If low-frequency components of the input signal are filtered out rather than high frequencies, the system is called a high-pass filter. The chemotaxis pathway is a band-pass filter (see Figure 2), filtering out low and high-frequency components.

To obtain a succinct measure for the signalling response due to an input concentration change $\Delta c(t)$, we define $\Delta R^{2}$ the integral of the response over frequency

$$
\Delta R^{2}=\frac{1}{2 \pi} \int_{-\infty}^{\infty} d \omega\left|\hat{\chi}_{R}(\omega) \Delta \hat{c}(\omega)\right|^{2} .
$$

\section{Noise}

Fluctuations $\delta R(t)$ around the mean value $\langle R(t)\rangle$ can be characterised by their correlations at different time points. The autocorrelation function $K$ is defined as

$$
K(\tau)=\langle\delta R(t) \delta R(t+\tau)\rangle,
$$

i.e. the average value of the product of fluctuations at two time points. It only depends on the interval between time points if the dynamics of $R(t)$ is stationary, i.e. the mean value $\langle R(t)\rangle$ and variance $\left\langle\delta R^{2}(t)\right\rangle$ do not vary with time $t$. Averaging over different measurements of $R(t)$ is indicated by angular brackets. Typically, correlations decay with the interval length $\tau$ between time points. 
Here, we use the power spectrum to characterise fluctuations. According to the Wiener-Kinchin theorem the power spectrum is the Fourier transform of the autocorrelation function [67],

$$
S_{R}(\omega)=\int_{-\infty}^{\infty} K(t) e^{i \omega t} d t .
$$

For exponentially decaying correlations as in Figure 2, the power spectrum is Lorentzian, i.e. has the frequency dependency

$$
S_{R}(\omega) \propto \frac{1}{\omega^{2}+\omega_{\alpha}^{2}} .
$$

The power spectrum can be calculated from the absolute square of the Fourier transform of time series $\delta R(t)$ measured or simulated over a time interval $T$,

$$
S_{R}(\omega)=\lim _{T \rightarrow \infty} \frac{\left\langle\delta \hat{R}(\omega) \delta \hat{R}^{*}(\omega)\right\rangle}{T},
$$

where the Fourier transform is defined on the finite measurement interval $T$ and the average $\langle\cdot\rangle$ is over multiple time series.

The variance of a stationary process can be calculated as the integral of the power spectrum over frequency,

$$
\left\langle\delta R^{2}\right\rangle=\frac{1}{2 \pi} \int_{-\infty}^{\infty} d \omega S_{R}(\omega) .
$$

\section{Noise sources}

\section{Determining the noise intensity}

Here, we use the Langevin approximation to describe fluctuations (noise) around average molecule numbers. For instance, consider the following general stochastic differential equation:

$$
\frac{d R}{d t}=r_{1}-r_{2}+\eta(t)
$$

The rates $r_{1}$ and $r_{2}$ typically depend on the concentrations of proteins in the signalling network. The noise term $\eta(t)$ is composed of two terms $\eta_{1}(t)$ and $\eta_{2}(t)$, which are associated with the rates $r_{1}$ and $r_{2}$, respectively. We assume $\eta_{1}$ and $\eta_{2}$ to be independent, i.e. $\left\langle\eta_{1}\right.$ (t) $\left.\eta_{2}\left(t^{\prime}\right)\right\rangle=0$. In general, this is justified as different reactions are catalysed by different proteins. Using $\left\langle\eta_{j}(t)\right.$ $\left.\eta_{j}\left(t^{\prime}\right)\right\rangle=Q_{j} \delta\left(t-t^{\prime}\right)$, the noise intensities can be calculated if we make the assumption that fluctuations are due to so-called birth and death processes, i.e. creation and destruction of the molecules with average rates $r_{1}^{*}$ and $r_{2}^{*}$. Then the associated noise intensities are $Q_{1}=r_{1}^{*}$ and $Q_{2}=r_{2}^{*}[8]$. The intensity of the total noise $\eta(t)$ is the sum $Q=Q_{1}+Q_{2}$ due to the independence of the two noises. As forward and backward rate are equal at steady state, $Q$ is twice the reaction rate in one direction at steady-state.

\section{Switching noise}

The switching noise $\eta_{A}(t)$ in Eq. 1 is due to the switching of each receptor complex between on and off states. We assume the switching to be a fast process, which can be described by the following dynamics of the receptor-complex on-bias $a$ :

$$
\frac{d a}{d t}=k_{1}(N-a)-k_{2} a+\eta_{a}(t) .
$$

The noise term $\eta_{a}(t)$ is a Gaussian white noise with zero mean and noise intensity $Q_{a}=2 k_{2} A^{*}$, where we used that the receptor complex activity $A=\langle a\rangle$ which is equal to the (quasi) steady-state activity of $a$, and $A=$ $A^{*}$ when adapted. The power spectrum of $a$ due to switching between on and off states is

$$
S_{a}(\omega)=\frac{Q_{a}}{\omega^{2}+\left(k_{1}+k_{2}\right)^{2}}
$$

where $k_{1}+k_{2}$ is the characteristic frequency of switching. Hence, the high-frequency component of fluctuations $\delta a(t)$ is reduced due to averaging by the finite rates of switching. Hence, the power spectrum of activity fluctuations $\eta_{A}(t)$, which are due to fluctuations in the rate of receptor complex switching $d a / d t$, is

$$
S_{\eta_{A}}(\omega)=\omega^{2} S_{a}(\omega) .
$$

This result is due to the Fourier transform of the rate of receptor complex switching $d a / d t$ yielding $i \omega \hat{a}$. Hence, the power spectra of $a$ and $d a / d t$ differ by a factor $\omega^{2}$ as the power spectrum is proportional to the magnitude squared of the Fourier transform according to Eq. 26.

\section{Ligand noise}

The number of ligand molecules in the vicinity of a receptor complex fluctuates due to binding/unbinding, and potential rebinding of previously bound ligand molecules at this complex, as well as diffusion $[57,68]$. Here, we use a simplified description of diffusion to calculate the spectrum of noise in the ligand dynamics $\eta_{c}$ $(t)$ in Eq. 2. Consider a volume whose dimensions are given by the diameter of a receptor complex $s=\sqrt{N} s_{R}$, where $s_{R}=1 \mathrm{~nm}$ is the size of a receptor dimer [69]. The change of ligand-molecule number $L$ in this volume is determined by the exchange rate $k_{D} \approx D /\left(2 s^{2}\right)$ due to diffusion [70]:

$$
\frac{d L}{d t}=k_{D}\left(c_{0} s^{3}-L\right)+\eta_{L}(t)
$$

where $k_{D} L$ is the rate of molecules moving out of the volume by diffusion, and $k_{D}$ times the mean 
concentration $c_{0}$ in solution serves as a proxy of the rate of ligand molecules moving into the volume. The noise term $\eta_{L}(t)$ is assumed to be Gaussian and white, with zero mean and noise intensity $Q_{L}=D s c_{0}$.

The power spectra of the number $L$ and concentration $c$ of molecules at receptor complex $j$ are respectively

$$
\begin{aligned}
& S_{L}(\omega)=\frac{D s c_{0}}{\omega^{2}+k_{D}^{2}} \\
& S_{c}(\omega)=\frac{S_{L}(\omega)}{s^{6}},
\end{aligned}
$$

where $s^{6}$ is the squared volume given by the dimension of the receptor complex. The zero-frequency limit of the power spectrum of the ligand concentration $S_{c}(0)$ $=c_{0} /(D s)$, which corresponds to calculations by Berg and Purcell [71] and Bialek and Setayeshgar [57] for the uncertainty in sensing ligand concentration.

The noise $\eta_{c}(t)$ in Eq. 2 is related to rate of change of the ligand concentration, similar to the considerations of the switching noise above. Hence, the power spectrum of the ligand fluctuations $\eta_{c}(t)$ is

$$
S_{\eta_{c}}(\omega)=\omega^{2} S_{c}(\omega) .
$$

\section{Methylation noise}

The size of fluctuations in the rate of methylation of a receptor complex $j$ in Eq. 3 is estimated from the average rates of methylation and demethylation at the adapted state, respectively. The noise $\eta_{M}(t)$ is assumed to be Gaussian and white, with zero mean, noise intensity $Q_{M}=2 \gamma_{R}\left(N-A^{*}\right)$ and power spectrum

$$
S_{\eta_{M}}(\omega)=Q_{M} .
$$

\section{Motor switching noise}

The noise in motor switching rate in Eq. 4 is assumed to be a Gaussian white noise term with zero mean, noise intensity $Q_{X}=2 k_{+}\left(A_{c}^{*}\right)\left(1-P_{C W}^{*}\right)$ and power spectrum

$$
S_{\eta_{X}}(\omega)=Q_{X} .
$$

\section{Calculation of response functions}

After linearising around the steady state and inserting the Fourier transforms we obtain for the simplified model

$$
\begin{aligned}
& -i \omega \Delta \hat{A}=-i \omega \frac{\partial A}{\partial M} \Delta \hat{M}-i \omega \frac{\partial A}{\partial c} \Delta \hat{c} \\
& -i \omega \Delta \hat{M}=-\omega_{1} \Delta \hat{A}
\end{aligned}
$$

$$
-i \omega \Delta \hat{P}_{C W}=\omega_{2} \Delta \hat{A}_{c}-\omega_{X} \Delta \hat{P}_{C W}
$$

where

$$
\omega_{1}=\gamma_{R}+3 \gamma_{B} A^{* 2}=\gamma_{R}\left(3-2 A_{r}^{*}\right) / A_{r}^{*}
$$

with $A^{*}=N \cdot A_{r}^{*} \approx N / 3[72]$ the adapted activity of a receptor complex, $A_{r}^{*}$ denoting the adapted activity of individual receptors. In the second equality we have used that at the adapted state $\gamma_{R}\left(N-A^{*}\right)=\gamma_{B} A^{* 3}$. The parameter $\omega_{2}=\left(1-P_{C W}^{*}\right) \frac{\partial k_{+}}{\partial A_{c}}-P_{C W}^{*} \frac{\partial k_{-}}{\partial A_{c}}$ is the derivative of the motor switching rates with respect to activity, and $\omega_{X}=k_{+}{ }^{*}+k_{-}^{*}$ is a characteristic frequency due to motor switching at steady state. $\Delta A$ is the response of every receptor signalling complex, and $\Delta A_{c}$ $=N_{C} \Delta A$ is the activity response of all receptor complexes in a cell. Solving for $\Delta \hat{A}_{c}$ and $\Delta \hat{P}_{C W}$, and division by the stimulus $\Delta \hat{c}$ yields the response functions in Eq. 5 and 6.

\section{Calculation of noise power spectra}

To calculate spectra, we linearise the deterministic parts of Eq. 1-4 similar to the calculation of the response functions, and formally Fourier transform the equations. We obtain

$$
\begin{aligned}
&-i \omega \delta \hat{A}_{c}=-i \omega \frac{\partial A}{\partial M} \sum_{j} \delta \hat{M}_{j}+\frac{\partial A}{\partial c} \sum_{j} \hat{\eta}_{c_{j}}+ \\
&+\sum_{j} \hat{\eta}_{A_{j}} \\
&-i \omega \delta \hat{M}_{j}=-\omega_{1} \delta \hat{A}_{j}+\hat{\eta}_{M_{j}} \\
&-i \omega \delta \hat{P}_{C W}=\omega_{2} \delta \hat{A}_{c}-\omega_{X} \delta \hat{P}_{C W}+\hat{\eta}_{X} .
\end{aligned}
$$

We solve for the Fourier transformed activity fluctuations $\delta \hat{A}_{c}$ and obtain

$$
\delta \hat{A}_{c}=\frac{\frac{\partial A}{\partial M} \sum_{j} \hat{\eta}_{M_{j}}+\frac{\partial A}{\partial c} \sum_{j} \eta_{c_{j}}+\sum_{j} \hat{\eta}_{A_{j}}}{\omega_{M}-i \omega}
$$

which yields the power spectrum in Eq. 8. The parameter $\omega_{M}=\omega_{1} \partial A / \partial M$, and we used Eq. 31 and 35 . From Eq. 44 we obtain for the Fourier transformed fluctuations in the motor bias $\delta \hat{P}_{C W}$

$$
\delta \hat{P}_{C W}=\frac{\omega_{2} \delta \hat{A}_{c}+\hat{\eta}_{X}}{\omega_{X}-i \omega}
$$

and their power spectrum is given by Eq. 9 . 


\section{Integrated signal response, variance and SNR Optimal receptor complex size}

The integrated response of the receptor activity to a step stimulus is

$$
\begin{aligned}
\Delta A_{c}^{2} & =\int_{-\infty}^{\infty} d \omega\left|\hat{\chi}_{A_{c}}(\omega) \Delta \hat{c}(\omega)\right|^{2} \\
& =\frac{\pi N_{C}^{2}\left(\frac{\partial A}{\partial c}\right)^{2}(\alpha c)^{2}}{\omega_{M}},
\end{aligned}
$$

where we inserted Eq. 5 . Hence, the activity response scales as $\Delta A_{c}^{2} \propto\left(N_{\text {tot }} / N\right)^{2}\left(N^{2}\right)^{2} / N \propto N$, where we used that $N_{C}=N_{\text {tot }} / N$ with $N_{\text {tot }}$ the total number of receptors in a cell.

The variance of the receptor activity is given by the integral of the power spectrum of activity fluctuations Eq. 8

$$
\begin{aligned}
\left\langle\delta A_{c}^{2}\right\rangle= & \frac{N_{C}}{2 \pi} \int_{-\tau^{-1}}^{\tau^{-1}} d \omega \frac{\omega^{2}\left[S_{a}(\omega)+\left(\frac{\partial A}{\partial c}\right)^{2} S_{c}(\omega)\right]}{\omega^{2}+\omega_{M}^{2}} \\
& +\frac{N_{C}}{2 \pi} \int_{-\tau^{-1}}^{\tau^{-1}} d \omega \frac{\left(\frac{\partial A}{\partial M}\right)^{2} Q_{M}}{\omega^{2}+\omega_{M}^{2}},
\end{aligned}
$$

where we consider the frequency range relevant for motor switching indicated by $\tau^{-1} \approx 0.1 \ldots 1 \mathrm{~Hz}$. The contribution from receptor switching is

$$
\left\langle\delta A_{c}^{2}\right\rangle_{a}=\frac{N_{C}}{2 \pi} \int_{-\tau^{-1}}^{\tau^{-1}} d \omega \frac{\omega^{2} S_{a}(\omega)}{\omega^{2}+\omega_{M}^{2}} \approx \frac{2 k_{2} A_{r}^{*} N_{t o t}}{\pi \tau\left(k_{1}+k_{2}\right)^{2}}
$$

where we used $Q_{a}$ and inserted Eq. 30 for the power spectrum of receptor switching noise and used that it is almost constant and equal to its zero-frequency value over the integration range. Furthermore, the factor $\omega^{2} /\left(\omega^{2}+\omega_{M}^{2}\right) \approx 1$ and $A_{r}^{*}=A^{*} / N$ is the adapted activity of an individual receptor. Hence, according to this simple calculation the contribution to the variance from receptor switching is roughly constant with receptor complex size.

The contribution from ligand diffusion is

$$
\begin{aligned}
\left\langle\delta A_{c}^{2}\right\rangle_{c} & =\frac{N_{C}}{2 \pi} \int_{-\tau^{-1}}^{\tau^{-1}} d \omega \frac{\omega^{2}\left(\frac{\partial A}{\partial c}\right)^{2} S_{c}(\omega)}{\omega^{2}+\omega_{M}^{2}} \\
& \approx N_{C}\left(\frac{\partial A}{\partial c}\right)^{2}\left\langle\delta c^{2}\right\rangle,
\end{aligned}
$$

where $\left\langle\delta c^{2}\right\rangle=c_{0} /(D s \tau)$ is the variance of the ligand concentration measured during the time interval $\tau$. We used Eq. 34 and the same argument as for the switching noise to calculate the integral. Hence, the contribution to the variance from the ligand diffusion grows as $\left\langle\delta A_{c}^{2}\right\rangle_{c} \propto N^{5 / 2}$ as a result of incoherent addition of noise from different receptor complexes, the sensitivity $\partial A / \partial c$ increasing as $N^{2}$, and the size dependence of receptor complexes $s \propto N^{1 / 2}$.

The contribution to the variance from receptor methylation is

$$
\begin{aligned}
\left\langle\delta A_{c}^{2}\right\rangle_{M} & =\frac{N_{C}}{2 \pi}\left(\frac{\partial A}{\partial M}\right)^{2} \int_{-\tau^{-1}}^{\tau^{-1}} d \omega \frac{Q_{M}}{\omega^{2}+\left(\omega_{1} \frac{\partial A}{\partial M}\right)^{2}} \\
& \approx \frac{2 N_{t o t} \gamma_{R}\left(1-A_{r}^{*}\right) A_{r}^{*}}{\omega_{1}} \frac{\partial A}{\partial M}
\end{aligned}
$$

where we defined $\omega_{1}=\gamma_{R}+3 \gamma_{B} N^{2}\left(A_{r}^{*}\right)^{2}$, inserted $Q_{M}=2 \gamma_{R} N\left(1-A_{r}^{*}\right)$ and $\omega_{M}=\omega_{1}(\partial A / \partial M)$. Hence, $\left\langle\delta A_{c}^{2}\right\rangle_{M}$ grows approximately linearly with receptor complex size.

The SNR grows linearly with $N$ for small complex sizes, and decreases as $N^{-2}$ for larger complex sizes, resulting in an optimal medium receptor complex size, in qualitative agreement with Figure 6.

\section{Optimal adaptation rates}

The integrated signal response of the receptor activity Eq. 47

$$
\Delta A_{c}^{2}=\frac{\pi N_{C}^{2}\left(\frac{\partial A}{\partial c}\right)^{2}(\alpha c)^{2}}{\omega_{1} \frac{\partial A}{\partial M}},
$$

where the numerator expresses the initial response of receptors of concentration changes and the denominator the filtering by adaptation. The sensitivity $\partial A / \partial c=N A_{r}^{*}\left(1-A_{r}^{*}\right) h(c)$, where $h(c)=\partial F / \partial c$, $\omega_{1}=\gamma_{R}+3 \gamma_{B} N^{2}\left(A_{r}^{*}\right)^{2}$, and $\partial A / \partial M=N A_{r}^{*}\left(1-A_{r}^{*}\right) / 2$. The adapted activity can be obtained analytically for our simplified model from the steady state of the methylation dynamics Eq. 3,

$$
A_{r}{ }^{*}=\sqrt[3]{\frac{1}{2} \beta+\sqrt{\frac{\beta^{2}}{4}+\frac{\beta^{3}}{27}}}-\frac{\beta}{3 \sqrt[3]{\frac{1}{2} \beta+\sqrt{\frac{\beta^{2}}{4}+\frac{\beta^{3}}{27}}}},
$$

and is only a function of the ratio $\beta=\gamma_{R} / \gamma_{B}$. Expanding the adapted activity around $A_{r}^{*}=0\left(\right.$ for $\left.\gamma_{R} \rightarrow 0\right)$ yields $A_{r}^{*} \propto \gamma_{R}^{1 / 3}$, and around $A_{r}^{*}=1$ (for $\gamma_{B} \rightarrow 0$ ) yields $A_{r}^{*} \propto \gamma_{B}$. Similarly, $\omega_{1} \propto \gamma_{R}^{2 / 3}$ (const. $\left.+\gamma_{B}^{4 / 3}\right)$. Hence, $\partial A / \partial c \propto \gamma_{R}^{1 / 3}\left(\gamma_{B}\right)$ and $\omega_{1} \partial A / \partial M \propto \gamma_{R}^{4 / 3}\left(\gamma_{B}\right)$.

The initial response to concentration changes decreases slower than adaptation times, resulting in an increased signal response for vanishing $\gamma_{R}$. For vanishing $\gamma_{B}$, the initial response to concentration changes decreases faster than adaptation speed, hence yielding a vanishing signal response. The overall dependence of the integrated signal response is $\Delta A_{c}^{2} \propto \gamma_{R}^{-1 / 3}\left(\gamma_{B}\right)$ for $\gamma_{R} \rightarrow 0\left(\gamma_{B} \rightarrow 0\right)$. For the contributions to the variance of the receptor activity from receptor switching, ligand diffusion and receptor 
Table 1 Parameters of the full pathway model, including references to literature

\begin{tabular}{lll}
\hline Parameter & Value & Reference \\
\hline$[A]_{\text {tot }}$ & $5 \mu \mathrm{M}$ & {$[73]$} \\
{$[B]_{\text {tot }}$} & $0.28 \mu \mathrm{M}$ & {$[49]$} \\
{$[R]_{\text {tot }}$} & $0.16 \mu \mathrm{M}$ & {$[49]$} \\
{$\left[Y_{\text {tot }}\right.$} & $9.7 \mu \mathrm{M}$ & {$[49]$} \\
$V_{\text {cell }}$ & $1.4 \mathrm{fl}$ & {$[73]$} \\
$N_{A, \text { tot }}$ & 4215 & calculated from above \\
$N_{B, \text { tot }}$ & 236 & calculated from above \\
$N_{R, \text { tot }}$ & 135 & calculated from above \\
$N_{Y, \text { tot }}$ & 8177 & calculated from above \\
$N_{A, \text { tot }}=N N_{C}$ & 7027 & {$[49,73]$} \\
$k_{2}$ & $10^{3} \mathrm{~s}^{-1}$ & {$[74]$} \\
$k_{A}$ & $10^{3} \mathrm{~s}^{-1}$ & {$[75]$} \\
$k_{y}$ & $100 \mu \mathrm{M}^{-1} \mathrm{~s}^{-1}$ & {$[76]$} \\
$k_{B}$ & $15 \mu \mathrm{M}^{-1} \mathrm{~s}^{-1}$ & {$[76]$} \\
$k_{-Y}$ & $5 \mathrm{~s}^{-1}$ & adjusted to yield steady-state value \\
$k_{-B}$ & $1.35 \mathrm{~s}^{-1}$ & $\left(0.35 \mathrm{~s}^{-1}\right)[77,78]$ \\
$\gamma_{R}$ & $0.0061 \mathrm{~s}^{-1}$ & {$[47]$} \\
$\gamma_{B}$ & $3.14 \mu \mathrm{M}^{-2} \mathrm{~s}^{-1}$ & {$[47]$} \\
\hline
\end{tabular}

The literature values are given in parentheses where different from our parameter values. $k_{-}$was determined by the condition that at steady-state with $A_{R}^{*}=1 / 3$, the concentration $\left[Y_{p}\right]^{*}=\left[Y_{\text {tot }} / 3[73]\right.$.

Table 2 Intensities of Gaussian white noise terms in the full pathway model

\begin{tabular}{lll}
\hline process & index $\boldsymbol{i}$ & noise intensity $Q_{i}$ \\
\hline receptor switching & $a$ & $2 k_{2} A^{*}$ \\
ligand diffusion & $L$ & $2 D s C_{0}$ \\
receptor de/methylation & $M$ & $2 \gamma_{R}\left(N-A^{*}\right)$ \\
CheA autophosphorylation & $A_{p}$ & $A_{c}^{*}\left(\frac{k_{A}}{N_{C} N}\right)\left(N_{A, t o t}-N_{A_{p}}^{*}\right)$ \\
CheY phosphorylation & $A_{,} Y_{p}$ & $\left(\frac{k_{y}}{V_{c e l l}}\right)\left(N_{Y, \text { tot }}-N_{Y_{p}}^{*}\right) N_{A_{p}}^{*}$ \\
CheB phosphorylation & $A_{,} B_{p}$ & $\left(\frac{k_{b}}{V_{c e l l}}\right)\left(N_{B, t o t}-N_{B_{p}}^{*}\right) N_{A_{p}}^{*}$ \\
CheY dephosphorylation & $-Y_{p}$ & $k_{-\gamma} N_{Y_{p}}^{*}$ \\
CheB dephosphorylation & $-B_{p}$ & $k_{-b} N_{B_{p}}^{*}$ \\
motor switching & $X$ & $2 k_{+}^{*} k_{-}^{*}$ \\
$k_{+}^{*}+k_{-}^{*}$
\end{tabular}

Index $i$ represents noise term $\eta$

methylation dynamics we obtain $\left\langle\delta A_{c}^{2}\right\rangle_{a} \propto \gamma_{R}^{1 / 3}\left(\gamma_{B}\right)$, $\left\langle\delta A_{c}^{2}\right\rangle_{M} \propto \gamma_{R}\left(\gamma_{B}^{2 / 3}\right)$ and $\left\langle\delta A_{c}^{2}\right\rangle_{M} \propto \gamma_{R}\left(\gamma_{B}^{2 / 3}\right)$, respectively. Hence, according to our simplified model the SNR of the receptor activity goes as $\mathrm{SNR} \propto \gamma_{R}^{-2 / 3}\left(\gamma_{B}^{4 / 3}\right)$, in qualitative agreement with Figure 7.
Table 3 Fitting parameters for response function of the full pathway model for Fig. 3

\begin{tabular}{|c|c|c|c|}
\hline \multirow[t]{2}{*}{ Parameter } & \multirow{2}{*}{$\begin{array}{l}\text { Block et al., Segall et al. } \\
{[26,27]\left[\mathrm{s}^{-1}\right]}\end{array}$} & \multicolumn{2}{|c|}{ Shimizu et al. [28] } \\
\hline & & $\begin{array}{l}32^{\circ} \mathrm{C} \\
{\left[\mathrm{s}^{-1}\right]}\end{array}$ & $\begin{array}{l}22^{\circ} \mathrm{C} \\
{\left[\mathrm{s}^{-1}\right]}\end{array}$ \\
\hline \multicolumn{4}{|l|}{ adaptation: } \\
\hline$\lambda_{1}(\partial A=\partial M)$ & 0.178 & 0.018 & 0.0039 \\
\hline$\lambda_{9}$ & 0.0263 & 0.0027 & $5.610^{-4}$ \\
\hline \multicolumn{4}{|c|}{ motor switching: } \\
\hline$\lambda_{7}$ & $4.410^{-4}$ & - & - \\
\hline$\lambda_{8}$ & 2.111 & - & - \\
\hline
\end{tabular}

\section{Numerical integration of different equations of the full model}

Only the full pathway model without noise was solved numerically (cf. Figure 4). We used an Euler method to integrate the differential equations 13-17 without noise. As concentration input signal we used a triangular impulse of duration $0.02 \mathrm{~s}, c_{0}=0$ and peak concentration $10^{-3} \mathrm{mM}$. The integration time step was $\Delta t=10^{-3}$, maximum integration time was $100 \mathrm{~s}$. In each integration step, first the receptor complex activity was calculated according to Eq. 11 based on the current ligand concentration and receptor methylation level from the previous iteration. All other variables $x$ were updated in each time step $i$ according to $x_{i+1}=x_{i}+\Delta t \cdot$ rate, where rate is given by the r.h.s. of the differential equations for the respective variable.

\section{Parameters}

Rate constants and total cell concentrations of proteins for the full pathway model are given in Table 1. The noise terms $\eta_{A_{j}}, \eta_{c_{j}}, \eta_{M_{j}}$ and $\eta_{X}$ are the same as in Eq. 24 and 27-29 and their power spectra are given there. The noise associated with phosphorylation and dephosphorylation $\eta_{A_{p}}, \eta_{A, B_{p}}, \eta_{A, Y_{p}}, \eta_{-B_{p}}$ and $\eta_{-Y_{p}}$ are assumed to be Gaussian white noise terms with zero mean and autocorrelations $\left\langle\eta_{i}(t) \eta_{i}\left(t^{\prime}\right)\right\rangle=Q_{i} \delta\left(t-t^{\prime}\right)$ with noise intensities

Table 4 Parameters for cell-to-cell variation in Fig. 5A

\begin{tabular}{lllll}
\hline Parameter & WT1 (black line) & red line & green line & blue line \\
\hline$k_{+}^{*}\left[\mathrm{~s}^{-1}\right]$ & 1.05 & 52.4 & 1.05 & 1.05 \\
$k_{-}^{*}\left[\mathrm{~s}^{-1}\right]$ & 1.06 & 53.0 & 1.06 & 1.06 \\
$\gamma_{R}\left[\mathrm{~s}^{-1}\right]$ & 0.0061 & 0.0061 & $6.110^{-5}$ & 0.0061 \\
$\gamma_{B}\left[\mu \mathrm{M}^{-2} \mathrm{~s}^{-1}\right]$ & 3.14 & 3.14 & $3.1410^{-2}$ & 3.14 \\
$N_{\text {tot }}$ & 7000 & 7000 & 7000 & 70 \\
\hline
\end{tabular}

For additional parameters see Table 1. 
Table 5 Parameters for cells with low motor bias in Fig. $5 B$ and $C$

\begin{tabular}{ll}
\hline Parameter & value \\
\hline$N_{\text {tot }}$ & 4200 \\
$Y_{\text {tot }}[\mu \mathrm{M}]$ & 2 \\
$k_{+}^{*}\left[\mathrm{~s}^{-1}\right]$ & 0.015 \\
$k_{-}^{*}\left[\mathrm{~s}^{-1}\right]$ & 4.75 \\
$\partial k_{+} / \partial Y_{p}\left[\mathrm{~s}^{-1} \mu \mathrm{M}^{-1}\right]$ & 4.75 \\
$\partial \mathrm{k}_{-} / \partial Y_{p}\left[\mathrm{~s}^{-1} \mu \mathrm{M}^{-1}\right]$ & -5.5 \\
\hline
\end{tabular}

For additional parameters see Table 1. Similar spectra with increased lowfrequency component have been found for cells with other motor biases [50].

Table 6 Parameters for the fluctuation-response theorem in Fig. 8

\begin{tabular}{llll}
\hline Parameter & $\begin{array}{l}\text { varying } \gamma_{\boldsymbol{R}} \\
\text { (solid) }\end{array}$ & $\begin{array}{l}\text { varying } \gamma_{B} \\
\text { (dashed) }\end{array}$ & $\begin{array}{l}\text { varying }[Y]_{\text {tot }} \\
\text { (dotted) }\end{array}$ \\
\hline$N_{\text {tot }}$ & 700 & 700 & 700 \\
$\gamma_{R}\left[\mathrm{~s}^{-1}\right]$ & varied & 0.0061 & 0.0061 \\
$\gamma_{B}\left[\mathrm{~s}^{-1}\right]$ & 3.14 & varied & 3.14 \\
{$\left[Y_{\text {tot }}[\mu \mathrm{M}]\right.$} & 9.7 & 9.7 & Varied \\
\hline
\end{tabular}

For additional parameters see Table 1.

$Q_{i}$ given in Table 2. Fitting parameters of the Fourier transformed response function Figure 3 are listed in Table 3. Parameters for Figure 5 are listed in Tables 4 and 5, and those for Figure 8 are listed in Table 6.

\section{Additional material}

Additional file 1: Supplementary information. Supplementary information in .pdf format, including the extended model for the chemotaxis pathway, additional mathematical derivations and validity checks.

\section{Acknowledgements}

We thank Richard Berry, Martin Buck, Tom Duke and William Ryu for helpful discussions, and Suhail Islam for computational support. RGE was supported by Biotechnological and Biological Sciences Research Council grant BB/ G000131/1 and the Centre for Integrative Systems Biology and Bioinformatics (CISBIO) at Imperial College.

\section{Author details}

'Division of Molecular Biosciences, Imperial College London, SW7 2AZ London, UK. ${ }^{2}$ Centre for Integrative Systems Biology and Bioinformatics, Imperial College London, UK. ${ }^{3}$ BioQuant, Universität Heidelberg, 69120 Heidelberg, Germany.

\section{Authors' contributions}

DC and RGE conceived and designed the study, performed analytical calculations, analysed the data, and wrote the paper. DC performed computer simulations. All authors read and approved the final manuscript.

Received: 24 March 2011 Accepted: 27 September 2011 Published: 27 September 2011
References

1. Sykulev Y, Joo M, Vturina I, Tsomides TJ, Eisen HN: Evidence that a Single Peptide MHC Complex on a Target Cell Can Elicit a Cytolytic T Cell Response. Immunity 1996, 4:565-571.

2. Mortimer D, Pujic Z, Vaughan T, Thompson AW, Feldner J, Vetter I, Goodhill GJ: Axon guidance by growth-rate modulation. Proc Natl Acad Sci USA 2010, 107:5202-5207.

3. Elowitz MB, Levine AJ, Siggia ED, Swain PS: Stochastic Gene Expression in a Single Cell. Science 2002, 297:1183-1186.

4. Pedraza JM, van Oudenaarden A: Noise propagation in gene networks. Science 2005, 307:1965-1969.

5. Paulsson J: Models of stochastic gene expression. Phys Life Rev 2005, 2:157-175.

6. Acar M, Pando BF, Arnold FH, Elowitz MB, van Oudenaarden A: A General Mechanism for Network-Dosage Compensation in Gene Circuits. Science 2010, 329:1656-1660.

7. Eldar A, Elowitz MB: Functional roles for noise in genetic circuits. Nature 2010, 467:167-173

8. Thattai M, van Oudenaarden A: Attenuation of noise in ultrasensitive signaling cascades. Biophys J 2002, 82:2943-2950.

9. Shibata T, Fujimoto K: Noisy signal amplification in ultrasensitive signal transduction. Proc Natl Acad Sci USA 2005, 102:331-336.

10. Colman-Lerner A, Gordon A, Serra E, Chin T, Resnekov O, Endy D, Pesce CG, Brent R: Regulated cell-to-cell variation in a cell-fate decision system. Nature 2005, 437:699-706.

11. Taylor RJ, Falconnet D, Niemistö A, Ramsey SA, Prinz S, Shmulevich I, Galitski T, Hansen CL: Dynamic analysis of MAPK signaling using a highthroughput microfluidic single-cell imaging platform. Proc Natl Acad Sci USA 2009, 106:3758-3763.

12. Detwiler PB, Ramanathan S, Sengupta A, Shraiman BI: Engineering aspects of enzymatic signal transduction: photoreceptors in the retina. Biophys $J$ $2000,79 \cdot 2801-2817$.

13. Hornung G, Barkai N: Noise Propagation and Signaling Sensitivity in Biological Networks: A Role for Positive Feedback. PLoS Comput Biol 2008 4:e8.

14. Lestas I, Vinnicombe G, Paulsson J: Fundamental limits on the suppression of molecular fluctuations. Nature 2010, 467:174-178.

15. Ueda M, Shibata T: Stochastic Signal Processing and Transduction in Chemotactic Response of Eukaryotic Cells. Biophys J 2007, 93:11-20.

16. Emonet T, Cluzel P: Relationship between cellular response and behavioral variability in bacterial chemotaxis. Proc Natl Acad Sci USA 2008, 105:3304-3309.

17. Mehta P, Goyal S, Wingreen NS: A quantitative comparison of sRNA-based and protein-based gene regulation. Mol Syst Biol 2008, 4:221.

18. Tostevin F, ten Wolde PR: Mutual Information between Input and Output Trajectories of Biochemical Networks. Phys Rev Lett 2009, 102:218101.

19. Mora T, Wingreen NS: Limits of Sensing Temporal Concentration Changes by Single Cells. Phys Rev Lett 2010, 104:248101.

20. Laub MT, Goulian M: Specificity in Two-Component Signal Transduction Pathways. Ann Rev Genet 2007, 41:121-145.

21. Berg HC: Motile behavior of bacteria. Phys Today 2000, 53:24-29.

22. Falke JJ, Hazelbauer GL: Transmembrane signaling in bacterial chemoreceptors. Trends Biochem Sci 2001, 26:257-265.

23. Sourjik V: Receptor clustering and signal processing in E. coli chemotaxis. Trends Microbiol 2004, 12:569-576.

24. Wadhams GH, Armitage JP: Making sense of it all: bacterial chemotaxis. Nat Rev Mol Cell Biol 2004, 5:1024-1037.

25. Baker MD, Wolanin PM, Stock JB: Systems biology of bacterial chemotaxis. Curr Opin Microbiol 2006, 9:187-192.

26. Block SM, Segall JE, Berg HC: Impulse responses in bacterial chemotaxis. Cell 1982, 31:215-226.

27. Segall JE, Block SM, Berg HC: Temporal comparisons in bacterial chemotaxis. Proc Natl Acad Sci USA 1986, 83:8987-8991.

28. Shimizu TS, Tu Y, Berg HC: A modular gradient-sensing network for chemotaxis in Escherichia coli revealed by responses to time-varying stimuli. Mol Syst Biol 2010, 6:382.

29. Block SM, Segall JE, Berg HC: Adaptation kinetics in bacterial chemotaxis. J Bacteriol 1983, 154:312-323.

30. Tu Y, Shimizu TS, Berg HC: Modeling the chemotactic response of Escherichia coli to time-varying stimuli. Proc Natl Acad Sci USA 2008 , 105:14855-14860. 
31. Korobkova E, Emonet T, Vilar J, Shimizu T, Cluzel P: From molecular noise to behavioral variability in a single bacterium. Nature 2004, 428:574-578.

32. Morton-Firth CJ, Bray D: Predicting temporal fluctuations in an intracellular signalling pathway. J Theor Biol 1998, 192:117-128.

33. Xing J, Bai F, Berry R, Oster G: Torque-speed relationship of the bacterial flagellar motor. Proc Natl Acad Sci USA 2006, 103:1260-1265.

34. Meacci G, Tu Y: Dynamics of the bacterial flagellar motor with multiple stators. Proc Natl Acad Sci USA 2009, 106:3746-3751.

35. Mora $T, Y u H$, Sowa $Y$, Wingreen NS: Steps in the bacterial flagellar motor. PLoS Comput Biol 2009, 5:e1000540.

36. Mora T, Yu H, Wingreen NS: Modeling torque versus speed, shot noise, and rotational diffusion of the bacterial flagellar motor. Phys Rev Lett 2009, 103:248102.

37. van Albada SB, Tănase-Nicola S, ten Wolde PR: The switching dynamics of the bacterial flagellar motor. Mol Syst Biol 2009, 5:316.

38. Scharf BE, Fahrner KA, Turner L, Berg HC: Control of direction of flagellar rotation in bacterial chemotaxis. Proc Natl Acad Sci USA 1998, 95:201-206.

39. Turner L, Samuel AD, Stern AS, Berg HC: Temperature dependence of switching of the bacterial flagellar motor by the protein CheY (13DK106YW). Biophys J 1999, 77:597-603.

40. Tu Y, Grinstein G: How White Noise Generates Power-Law Switching in Bacterial Flagellar Motors. Phys Rev Lett 2005, 94:208101.

41. van Kampen NG: Stochastic processes in physics and chemistry. North Holland 2007.

42. Sourjik V, Berg HC: Functional interactions between receptors in bacterial chemotaxis. Nature 2004, 428:437-441.

43. Mello BA, Tu Y: An allosteric model for heterogeneous receptor complexes: understanding bacterial chemotaxis responses to multiple stimuli. Proc Natl Acad Sci USA 2005, 102:17354-17359.

44. Keymer JE, Endres RG, Skoge M, Meir Y, Wingreen NS: Chemosensing in Escherichia coli: two regimes of two-state receptors. Proc Natl Acad SCi USA 2006, 103:1786-1791.

45. Endres RG, Wingreen NS: Precise adaptation in bacterial chemotaxis through "assistance neighborhoods". Proc Natl Acad Sci USA 2006, 103:13040-13044.

46. Endres RG, Oleksiuk $\mathrm{O}$, Hansen $\mathrm{CH}$, Meir $\mathrm{Y}$, Sourjik V, Wingreen NS: Variable sizes of Escherichia coli chemoreceptor signaling teams. Mol Syst Biol 2008, 4:211.

47. Clausznitzer D, Oleksiuk O, Sourjik V, Endres RG: Chemotactic response and adaptation dynamics in Escherichia coli. PLoS Comput Biol 2010, 6: e1000784

48. Stock G, Ghosh K, Dill KA: Maximum Caliber: A variational approach applied to two-state dynamics. J Chem Phys 2008, 128:194102.

49. Li M, Hazelbauer GL: Cellular Stoichiometry of the Components of the Chemotaxis Signaling Complex. J Bacteriol 2004, 186:3687-3694.

50. Park H, Pontius W, Guet CC, Marko JF, Emonet T, Cluzel P: Interdependence of behavioural variability and response to small stimuli in bacteria. Nature 2010, 468:819-823.

51. Korobkova E, Emonet T, Park H, Cluzel P: Hidden stochastic nature of a single bacterial motor. Phys Rev Letters 2006, 96:58105.

52. Blat Y, Gillespie B, Bren A, Dahlquist FW, Eisenbach M: Regulation of phosphatase activity in bacterial chemotaxis. J Mol Biol 1998, 284:1191-1199.

53. Blat $Y$, Eisenbach M: Oligomerization of the Phosphatase CheZ Upon Interaction with the Phosphorylated Form of CheY. J Biol Chem 1996, 271:1226-1231.

54. Blat $Y$, Eisenbach M: Mutants with Defective Phosphatase Activity Show No Phosphorylation-dependent Oligomerization of CheZ. J Biol Chem 1996, 271:1232-1236.

55. Eisenbach M: Bacterial chemotaxis. In Chemotaxis. Edited by: Eisenbach M Imperial College Press; 2004:53-215.

56. Hansen CH, Endres RG, Wingreen NS: Chemotaxis in Escherichia coli: a molecular model for robust precise adaptation. PLoS Comput Biol 2008, 4: e1.

57. Bialek W, Setayeshgar S: Physical limits to biochemical signaling. Proc Natl Acad Sci USA 2005, 102:10040-10045.

58. Aquino G, Clausznitzer D, Tollis S, Endres RG: Optimal receptor-cluster size determined by intrinsic and extrinsic noise. Phys Rev E 2011, 83:021914.

59. Sourjik V, Armitage JP: Spatial organization in bacterial chemotaxis. EMBO J 2010, 29:2724-2733.
60. Hutchings MI, Hong HJ, Buttner MJ: The vancomycin resistance VanRS two-component signal transduction system of Streptomyces coelicolor. Mol Microbiol 2006, 59:923-35

61. Henke JM, Bassler BL: Three parallel quorum-sensing systems regulate gene expression in Vibrio harveyi. J Bacteriol 2004, 186:6902-6914.

62. Jiang M, Shao W, Perego M, Hoch JA: Multiple histidine kinases regulate entry into stationary phase and sporulation in Bacillus subtilis. Mol Microbiol 2000, 38:535-342.

63. Porter SL, Wadhams GH, Armitage JP: Rhodobacter sphaeroides: complexity in chemotactic signalling. Trends in Microbiology 2008, 16:251-260.

64. Glekas GD, Cates JR, Cohen TM, Rao C, Ordal GW: Site-specific methylation in Bacillus subtilis chemotaxis: effect of covalent modifications to the chemotaxis receptor McpB. Microbiology 2011, 157:56-65.

65. Cluzel $P$, Surette $M$, Leibler S: An ultrasensitive bacterial motor revealed by monitoring signaling proteins in single cells. Science 2000 287:1652-1655.

66. Kubo R: Statistical Mechanical Theory of Irreversible Processes I. Journal of the Physical Society of Japan 1957, 12:570-586.

67. Stratonovich R: Topics in the Theory of Random Noise, Volume I New York: Gordon and Breach; 1963.

68. Endres RG, Wingreen NS: Accuracy of direct gradient sensing by cellsurface receptors. Prog Biophys Mol Biol 2009, 100:33-39.

69. Hazelbauer GL: Bacterial chemoreceptors. Curr Opin Struct Biol 1992, 2:505-510

70. Berg HC: Random Walks in Biology Princeton University Press, Princeton 1993 chap. Diffusion: Macroscopic Theory.

71. Berg HC, Purcell EM: Physics of chemoreception. Biophys J 1977, 20:193-219.

72. Sourjik V, Berg HC: Receptor sensitivity in bacterial chemotaxis. Proc Natl Acad Sci USA 2002, 99:123-127.

73. Sourjik V, Berg HC: Binding of the Escherichia coli response regulator CheY to its target measured in vivo by fluorescence resonance energy transfer. Proc Natl Acad Sci USA 2002, 99:12669-12674.

74. Shapovalov G, Lester HA: Gating Transitions in Bacterial lon Channels Measured at 3 us Resolution. J Gen Phys 2004, 124:151-161.

75. Wolanin PM, Baker MD, Francis NR, Thomas DR, DeRosier DJ, Stock JB: Selfassembly of receptor/signaling complexes in bacterial chemotaxis. Proc Natl Acad Sci USA 2006, 103:14313-14318.

76. Stewart RC, Jahreis K, Parkinson JS: Rapid phosphotransfer to CheY from a CheA protein lacking the CheY-binding domain. Biochemistry 2000, 39:13157-13165.

77. Bray D, Bourret RB: Computer analysis of the binding reactions leading to a transmembrane receptor-linked multiprotein complex involved in bacterial chemotaxis. Mol Biol Cell 1995, 6:1367-1380.

78. Stewart RC: Activating and inhibitory mutations in the regulatory domains of the methylesterase in bacterial chemotaxis. J Biol Chem 1993, 268:1921-1930

doi:10.1186/1752-0509-5-151

Cite this article as: Clausznitzer and Endres: Noise characteristics of the Escherichia coli rotary motor. BMC Systems Biology 2011 5:151.

\section{Submit your next manuscript to BioMed Central and take full advantage of:}

- Convenient online submission

- Thorough peer review

- No space constraints or color figure charges

- Immediate publication on acceptance

- Inclusion in PubMed, CAS, Scopus and Google Scholar

- Research which is freely available for redistribution

Submit your manuscript at www.biomedcentral.com/submit
C Biomed Central 